\title{
Acetylation of conserved lysines fine-tune mitochondrial malate dehydrogenase activity in land plants
}

Manuel Balparda ${ }^{1 *}$, Marlene Elsässer ${ }^{2,3^{*}}$, Mariana B. Badia ${ }^{4,5^{*}}$, Jonas Giese ${ }^{6}$, Anastassia Bovdilova ${ }^{4}$, Meike Hüdig ${ }^{1,4}$, Lisa Reinmuth ${ }^{2}$, Markus Schwarzländer ${ }^{3}$, Iris Finkemeier ${ }^{6 \#}$, Mareike SchallenbergRüdinger ${ }^{2 \#}$, Veronica G. Maurino, ${ }^{1, \#}$

${ }^{1}$ Molecular Plant Physiology, Institute of Molecular Physiology and Biotechnology of Plants (IMBIO), University of Bonn, Kirschallee 1, 53115 Bonn, Germany.

${ }^{2}$ Molecular Evolution, Institute for Cellular and Molecular Botany (IZMB), University of Bonn, Kirschallee 1, 53115 Bonn, Germany

${ }^{3}$ Plant Energy Biology, Institute of Plant Biology and Biotechnology (IBBP), University of Münster, Schlossplatz 8, 48143 Münster, Germany

${ }^{4}$ Plant Molecular Physiology and Biotechnology, Institute of Developmental and Molecular Biology of Plants, Heinrich Heine University, and Cluster of Excellence on Plant Sciences (CEPLAS), Düsseldorf, Germany.

${ }^{5}$ Instituto de Biología Molecular y Celular de Rosario (IBR-CONICET), Universidad Nacional de Rosario, 2000 Rosario, Argentina.

${ }^{6}$ Plant Physiology, Institute of Plant Biology and Biotechnology (IBBP), University of Münster, Schlossplatz 7, 48149 Münster, Germany

* These authors contributed equally to the work

\# Corresponding authors: Veronica G. Maurino (vero.maurino@uni-bonn.de), Mareike SchallenbergRüdinger (mrueding@uni-bonn.de) and Iris Finkemeier (iris.finkemeier@uni-muenster.de)

Author for contact: Veronica G. Maurino (vero.maurino@uni-bonn.de)

Running head: Acetylation of conserved lysines fine-tune metabolism 


\begin{abstract}
Plants need to rapidly and flexibly adjust their metabolism to changes of their immediate environment. Since this necessity results from the sessile lifestyle of land plants, key mechanisms for orchestrating central metabolic acclimation are likely to have evolved early. Here, we explore the role of lysine acetylation as a posttranslational modification to directly modulate metabolic function. We generated a lysine acetylome of the moss Physcomitrium patens and identified 638 lysine acetylation sites, mostly found in mitochondrial and plastidial proteins. A comparison with available angiosperm data pinpointed lysine acetylation as a conserved regulatory strategy in land plants. Focusing on mitochondrial central metabolism, we functionally analyzed acetylation of malate dehydrogenase $(\mathrm{mMDH})$, which acts as a hub of plant metabolic flexibility. In $P$. patens mMDH1, we detected a single acetylated lysine located next to one of the four acetylation sites detected in Arabidopsis thaliana mMDH1. We assessed the kinetic behavior of recombinant A. thaliana and P. patens mMDH1 with site-specifically incorporated acetyl-lysines. Acetylation of A. thaliana mMDH1 at K169, K170, and K334 decreases its oxaloacetate reduction activity, while acetylation of P. patens mMDH1 at K172 increases this activity. We found modulation of the malate oxidation activity only in A. thaliana mMDH1, where acetylation of K334 highly activated it. Comparative homology modelling of $\mathrm{MDH}$ proteins revealed that evolutionarily conserved lysines serve as hotspots of acetylation. Our combined analyses indicate lysine acetylation as a common strategy to fine-tune the activity of central metabolic enzymes with likely impact on plant acclimation capacity.
\end{abstract}

Keywords: protein acetylation, post-transcriptional regulation, mitochondria, metabolism, malate dehydrogenase.

Significance statement: We explore the role of lysine acetylation as a mechanism to directly modulate mitochondrial metabolism in land plants by generating the lysine acetylome of the moss Physcomitrium patens and comparing with available angiosperm data. We found acetylation of evolutionarily conserved lysines as a strategy to fine-tune the activity of mitochondrial malate dehydrogenase in a species-dependent molecular context.

\title{
Introduction
}

Flux through central metabolism is particularly dynamic in plants and can change dramatically in response to changes in external conditions or intrinsic demands. For instance, the tricarboxylic acid (TCA) cycle, which is localized in the mitochondria, can operate in a cyclic or non-cyclic mode depending on cell type and the demands for reducing power, ATP, and carbon skeletons (Sweetlove et al., 2010). Although glycolysis-derived pyruvate decarboxylation is considered to be the major entry 
point for carbon into the TCA cycle in most organisms, in green plant tissues it is malate rather than pyruvate that acts as the main substrate for the TCA cycle under most circumstances (Sweetlove et al., 2010). Mitochondrial malate dehydrogenase (mMDH; EC 1.1.1.37) interconverts malate and oxaloacetate (OAA) using $\mathrm{NAD}^{+} / \mathrm{NADH}$ as co-substrate. In vivo, the direction of the reaction depends on the demands of the cells and the redox state of the NAD pool in the matrix (Fig. 1). Apart from its classical role in supporting the TCA cycle flux to generate OAA from malate for respiration, mMDH provides OAA for the synthesis of aspartate, and - indirectly - of citrate as precursor for nitrogen assimilation (Fig. 1) (Hanning and Heldt, 1993, Sweetlove et al., 2010). In particular in illuminated leaves, $\mathrm{mMDH}$ can also support metabolic flux in the opposite direction to oxidize NADH to NAD ${ }^{+}$ for consumption by the photorespiratory glycine decarboxylase (Journet et al., 1981), and as part of the mitochondrial malate valve that connects the redox states of the NAD pools of the mitochondrial matrix and the cytosol through the exchange of malate and OAA (Fig. 1) (Scheibe, 2004, Scheibe et al., 2005, Sweetlove et al., 2010). All these aspects of mitochondrial metabolism are supported by models obtained through diel flux balance analysis (Shameer et al., 2019).

Two isoforms, mMDH1 (AT1G53240) and mMDH2 (AT3G15020), are expressed in A. thaliana, of which mMDH1 is the main isoform in green tissue (Tomaz et al., 2010, Hüdig et al., 2015). While the in vivo mMDH oligomerization state in plants is not yet definitively established, pig heart mMDH is active as a homodimer of $\sim 70 \mathrm{kDa}$, possessing two equivalent binding sites (Murphey et al., 1967, Noyes et al., 1974, Shore and Chakrabarti, 1976, Gleason et al., 1994). Mitochondrial malate is not only metabolized through $\mathrm{mMDH}$, but also via the NAD-dependent malic enzyme (NAD-ME) (Tronconi et al., 2008, Sweetlove et al., 2010, Maurino and Engqvist, 2015, Tronconi et al., 2020). Both enzymes together provide a remarkable degree of flexibility to plant respiratory metabolism, since they are able to supply mitochondrial carbon metabolism with substrate to respire, but also replenish the TCA cycle with carbon skeletons to maintain its function even when carbon skeletons are withdrawn for biosynthesis, e.g., of amino acids (Sweetlove et al., 2010). While the positioning of $\mathrm{mMDH}$ at a branchpoint of central carbon metabolism of plants and its variable engagement depending on flux mode makes active regulation of mMDH activity particularly likely, relatively little is known about this regulation. Modulation of $\mathrm{mMDH}$ activity is not only critical to direct carbon flux either to OAA or pyruvate, but it also has the potential to set the total (photo-) respiratory flux by $\mathrm{NAD}^{+}$provisioning to glycine decarboxylase; such modulation can additionally uncouple the redox states of the NAD pools of the matrix and the cytosol (Sweetlove et al., 2010, Shameer et al., 2019). Plant mMDH was not found to be redox-regulated through the matrix thioredoxin system. Instead, it is most probably controlled in response to variations in the matrix adenine nucleotide balance, as its in vitro activity is lowered by ATP and inhibited by an increase in the ATP/ADP ratio within the physiological range (Yoshida and Hisabori, 2016). Recently, in A. thaliana seedlings growing in liquid cultures and harvested at the beginning of the light period, mMDH1 was found to be acetylated at four 
different lysine residues: K170, K325, K329, and K334 (König et al., 2014a). However, the functional significance of those modifications has not been explored.

Posttranslational modifications (PTMs), such as phosphorylation and acetylation, can alter protein functions by affecting protein interactions, subcellular localization, or enzymatic activities (Matsuzaki et al., 2005, Yang and Seto, 2008, Ventura et al., 2010, Inuzuka et al., 2012, Bovdilova et al., 2019). Protein acetylation of lysine residues has long been recognized as a regulator of transcriptional control (Allfrey et al., 1964). More recently, this PTM emerged as a regulator of cellular metabolism and signaling in different organisms (Wang et al., 2010, Zhao et al., 2010, Finkemeier et al., 2011, Choudhary et al., 2014, König et al., 2014a).

Lysine acetylation has been found to be particularly widespread in bacterial and mitochondrial proteomes (Xu et al., 2009, Weinert et al., 2011). Two coenzymes of energy metabolism, acetyl-CoA and $\mathrm{NAD}^{+}$, are required as substrates for the reversible acetylation of lysine residues (Imai et al., 2000, Lin et al., 2012). Acetyl-CoA is the coenzyme of lysine acetyltransferases but can also acetylate proteins non-enzymatically at a pH higher than eight (Guan and Xiong, 2011, Hirschey et al., 2011, König et al., 2014a). $\mathrm{NAD}^{+}$is used by sirtuin-type deacetylases, which also reside within mitochondria of mammals (Imai et al., 2000) and plants (König et al., 2014b). In mitochondria, acetyl$\mathrm{CoA}$ and $\mathrm{NAD}^{+}$play key roles as metabolic regulators. While acetyl-CoA is produced in the mitochondrial matrix by the pyruvate dehydrogenase complex (PDC) and is oxidized in the TCA cycle, $\mathrm{NAD}^{+}$is required as electron acceptor in the TCA cycle. Respiration rates can have a major impact on protein acetylation, since changes in physiological $\mathrm{NAD}^{+}$concentration correlate with the activity of sirtuins (Lombard et al., 2007, Anderson et al., 2017). The mitochondrial acetylome of 10day-old Arabidopsis thaliana seedlings revealed 120 lysine-acetylated proteins, which contained a total of 243 lysine-acetylated sites (König et al., 2014a). Most TCA cycle enzymes of A. thaliana were found to be acetylated, which parallels findings in animals and bacteria (Wang et al., 2010, Masri et al., 2013, König et al., 2014a). That PDC and most of the TCA cycle enzymes were found to be lysine-acetylated across different organisms suggests that acetylation might represent an evolutionary conserved regulation system for TCA cycle function (Hosp et al., 2017).

Lysine acetylation is over-represented in both mitochondria and chloroplasts of angiosperm species, suggesting a prominent role of lysine acetylation in the direct modulation of the function of the endosymbiotic organelles (Hartl et al., 2017, Moller et al., 2020). Little is known about the conservation of acetylated lysines in distant land plant species, as investigations of lysine acetylation hitherto covered only flowering plant species (Melo-Braga et al., 2012, König et al., 2014a, SmithHammond et al., 2014, Fang et al., 2015, He et al., 2016, Zhen et al., 2016, Jiang et al., 2018).

Here, we used the model moss Physcomitrium (Physcomitrella) patens as an early branching land plant (Rensing et al., 2020) to devise a comparative proteomic analysis to shed light on the evolutionary conservation of lysine acetylation in mitochondrial proteins and its potential functional 
diversification in plants. Our analysis identified lysine acetylation of different mitochondrial proteins of $P$. patens, including $\mathrm{mMDH}$. Using $\mathrm{mMDH}$ as a model enzyme of central metabolic importance, we address the question of whether lysine acetylation represents an evolutionary conserved strategy to modulate mMDH activity. We synthesized A. thaliana and P. patens acetylated mMDH1 proteoforms at the identified lysine acetylation positions using the genetic code expansion strategy (Neumann et al., 2008), and assessed the kinetic behavior of the recombinant enzyme variants. Our results indicate acetylation of conserved lysines as a common strategy to modulate mitochondrial carboxylic acid metabolism by fine tuning mMDH activity.

\section{Results}

\section{Identification of mitochondrial lysine-acetylated proteins in $P$. patens}

To identify lysine-acetylated proteins of the moss P. patens, we analyzed its proteome via acetyllysine enrichment and liquid chromatography mass spectrometry (LC-MS/MS). The proteome was obtained from gametophores, which represent the haploid and dominant growth stage of mosses, analogous to the sporophyte growth stage of vascular plants (Strotbek et al., 2013). Overall, we detected 6428 protein groups, of which 638 were found to be acetylated on at least one and at most nine lysines (Fig. 2, Supporting Table S1).

We next classified the identified proteins according to their subcellular localization, making use of a previous analysis of the mitochondrial and plastid proteomes of P. patens (Müller et al., 2014). Additional proteins were grouped as organelle-localized when classified as such in the most recent annotation (Lang et al., 2018). In total, 1454 proteins were categorized as organellar proteins, 331 of which were acetylated (Fig. 2). Of those, 272 were annotated as plastidial proteins and 98 as mitochondrial, including 39 proteins that were classified as plastidial and/or mitochondrial (Supporting Table S2 and S3). A post-hoc manual inspection suggested that 16 out of the 98 putative mitochondrial proteins were in fact plastidial (11) or peroxisomal (5; Supporting Table S2), and the putative plastidial citrate synthase proteins were rather peroxisomal due to highest sequence similarity (65-74\%) to the peroxisomal citrate synthases of A. thaliana. Correcting these mis-classifications results in 52 mitochondrial, 30 mitochondrial or plastidial, and 242 plastidial proteins identified as lysine acetylated (Fig. 2).

Similar to previous findings in angiosperms (Hartl et al., 2017; Moller et al., 2020), lysine acetylation is particular abundant in the mitochondria and plastids of P. patens gametophores. We found that $20 \%$ of the identified mitochondrial proteins and $23 \%$ of the identified plastidial proteins were acetylated, while only $6 \%$ of the other identified proteins possessed this PTM (Fig. 2; Supporting Table S2). This observation mirrors previous results from isolated mitochondria of A. thaliana seedlings, where 120 acetylated protein groups were identified (König et al., 2014a). In P. patens, we observed lysine acetylation in most TCA cycle enzymes detected in the dataset (aconitase, NAD- and NADP- 
dependent isocitrate dehydrogenase, 2-oxoglutarate dehydrogenase, and $\mathrm{mMDH}$ ), which also mirrors previous findings in A. thaliana.

\section{Different lysine residues are acetylated in TCA cycle enzymes of A. thaliana and $P$. patens}

The specific sites of lysine acetylation in TCA cycle enzymes and other mitochondrial proteins were compared between orthologous proteins of P. patens and A. thaliana (König et al., 2014a). Proteins were clustered into orthologous groups, each consisting of one up to six different proteins per species, depending on the number of paralogous proteins encoded in the two genomes and detected in the datasets (Supporting Table S1, Table 1). The PDC E1 $\alpha$ as well as four of the identified TCA cycle enzymes are acetylated in both $P$. patens and A. thaliana (Table 1). In contrast, lysine acetylation of NADP isocitrate dehydrogenase proteins was detected in P. patens only (Supporting Table S1).

Altogether, we found acetylation of 22 different lysines in the TCA cycle enzymes and the E1 $\alpha$ subunit of the PDC in P. patens or A. thaliana. In 14 of the 22 positions, the lysine, acetylated in one species, was conserved in at least one orthologous protein of the other species. Only the E1 $\alpha$ subunit of the PDC and the aconitase of both species shared a lysine that was acetylated in both datasets (Table 1). A similar picture emerged for the other mitochondrial orthologous protein groups investigated (Supporting Table S4).

Orthologous proteins of A. thaliana and P. patens showed conserved lysine positions, but for each individual position, lysine acetylation was typically detected in one of the two species only (Table 1, Supporting Table S4).

In addition to the TCA cycle enzymes and the PDC, we classified 25 mitochondrial A. thaliana proteins and 31 mitochondrial $P$. patens proteins into 17 further orthologous groups. We found 99 lysine residues to be acetylated in at least one protein of these additional orthologous groups. 66 of these 99 positions $(67 \%)$ carried a lysine in orthologous proteins of the respective other species as well; $12(12 \%)$ of these conserved lysines were acetylated in orthologous proteins of both species (Supporting Table S4). Mitochondrial orthologous proteins of A. thaliana and P. patens showed 66\% conserved lysine positions ( 80 positions/121 positions), which were found to be acetylated in at least one of the orthologs compared in this study. For each individual position, however, lysine acetylation was typically detected in one of the two species only (Table 1, Supporting Table S4).

A. thaliana mMDH was found acetylated on four lysines (K170, K325, K329, and K334) (König et al., 2014a), while P. patens mMDH was acetylated in one unique lysine (K172 of Pp3c4_20940V3.1, in the following named mMDH1, or K170 of Pp3c12_8120V3.1, in the following named mMDH2) corresponding to K169 of A. thaliana mMDH1 (Table 1, Fig. 3). Acetylation at K170 of A. thaliana mMDH proteins and $\mathrm{K} 172$ of $P$. patens $\mathrm{mMDH}$ proteins could not be unequivocally assigned to one of the two paralogs, mMDH1 and mMDH2, present in each species (Supporting Fig. S1). However, in 
the proteomic datasets of both $A$. thaliana and P. patens, mMDH1 was more abundant than mMDH2 (Supporting Table S1, König et al. 2014). Hence, we focused our further analyses on the dominant $\mathrm{mMDH}$ protein, $\mathrm{mMDH} 1$, of both species.

\section{Lysine acetylation of mMDH along the phylogeny of land plants}

To analyse if acetylation of mMDH is evolutionarily conserved across land plants, we inspected available acetylome datasets of nine additional flowering plant species for the presence of acetylation sites in mMDH (Supporting Table S5). In six of these species, unique acetylated peptides of mMDHs were detected (Fig. 3, Supporting Fig. S1, Supporting Table S5). The species with available information on mMDH acetylation cover six different orders of flowering plants (A. thaliana: Brassicales (König et al. 2014), Fragaria ananassa: Rosales (Fang et al., 2015), Pisum sativum: Fabales (Smith-Hammond et al. 2014), Vitis vinifera: Vitales (Melo-Braga et al., 2012), Camellia sinensis: Ericales (Jiang et al. 2018), Oryza sativa and Brachypodium distachyon: Poales (Zhang et al., 2015, He et al., 2016, Xiong et al., 2016, Zhen et al., 2016, Zhou et al., 2018)).

We aligned the mMDH protein sequences of these six species with mMDHs of model plants representing different main land plant clades (Ferns: Azolla filiculoides and Salvinia cucullata; hornworts: Anthoceros agrestis, liverworts: Marchantia polymorpha and green algae: Chlamydomonas reinhardtii) including $P$. patens as model moss and $A$. thaliana as model flowering plant. Sequences were identified via Blast using A. thaliana mMDH1 as query. For selected model species, we identified $\mathrm{MDH}$ proteins clustering together with $\mathrm{mMDH}$ of A. thaliana and P. patens in phylogenetic analyses (Supporting Fig. S1). Peroxisomal and chloroplastic MDHs of $A$ thaliana and P. patens clustered in separate clades. For comparison, we included the E. coli $\mathrm{MDH}$ and human mMDH2 along with their known lysine acetylation sites (Zhao et al., 2010, Schilling et al., 2015) in the sequence alignment (Fig. 3). Sequences in the alignment are clustered based on the land plant phylogeny (Qiu et al., 2006, Bremer et al., 2009). Only for Oryza sativa, different acetylation sites in both mMDH paralogs were identified, and thus both proteins were included in the alignment (Zhao et al., 2010, He et al., 2016) (Fig. 3).

An interesting pattern emerges for the two neighboring lysine residues in the mMDH sequences that correspond to K169 and K170 in the A. thaliana sequence (Fig. 3). In different studies, these lysine residues were found acetylated in mMDHs of several plant species. K172 of P. patens mMDH1 (corresponding to K169 of A. thaliana), identified with high coverage in our P. patens acetylome, was also acetylated in mMDH1 of Camellia (Jiang et al., 2018) and Brachypodium (Zhen et al., 2016) leaves, and in mMDH2 of $O$. sativa leaves (Zhou et al., 2018). K170 of A. thaliana mMDH1 was also acetylated in mMDH1 of strawberry leaves (Fang et al., 2015) and grape vine exocarp (Melo-Braga et al., 2012), and in mMDH2 of O. sativa embryos (He et al., 2016) (Fig. 3). In rice mMDH2, the lysines 
at position 169 and 170 were identified as differentially acetylated in different organs. The other angiosperms in this dataset showed acetylation only on one or the other lysine (Fig. 3).

Interestingly, at positions corresponding to K169 and K170 in A. thaliana, all other investigated species show either a lysine or an arginine; the latter is similar in structure and chemical properties to the non-acetylated lysine. Even in the plastidial and peroxisomal MDHs of A. thaliana and P. patens, either a lysine or an arginine is present at these two positions (Supporting Table S4). The peroxisomal MDH1 of A. thaliana shares the acetylation site with mMDH1 of $P$. patens (Supporting Fig. S1, Supporting Table S4, (König et al., 2014a)). In the E. coli MDH and human MDH, however, position 169 is occupied by an asparagine, which would partially mimic the constantly acetylated lysine. Other acetylated lysines in A. thaliana mMDH1 are K325, K329, and K334 (König et al., 2014a), each of which was found to be acetylated in at least one other angiosperm species. Acetylated K325 was detected in V. vinifera, while acetylated $\mathrm{K} 329$ and $\mathrm{K} 334$ were found in O. sativa mMDH2 and mMDH1, respectively (Fig. 3). In addition, K329 was identified to be acetylated in E. coli MDH (Fig. 3).

\section{Enzymatic properties of site-specific acetylated A. thaliana and P. patens mMDH1 proteoforms}

The conservation of lysine acetylation sites in plant mMDH (Fig. 3) prompted us to investigate the influence of acetylation on the enzymatic properties of the enzyme in the model plants A. thaliana and $P$. patens. We selectively incorporate acetyl-lysine (AcK) at the lysines K170, K325, K329, and K334 of mMDH1 of A. thaliana (König et al., 2014a) as well as K172 of mMDH1 of P. patens (Table 1, Fig. 3, Supporting Tables S2 and S3). The lysine acetylation was incorporated into recombinantly expressed proteins using the amber suppression system in E. coli, which allows the co-translational addition of $\mathrm{N}$-acetyl lysine in response to a stop codon at the desired positions (Neumann et al., 2008, Neumann et al., 2009). A thaliana and $P$. patens non-modified mMDH1 and the protein versions carrying the single acetylated lysines were expressed in E. coli in the presence of $\mathrm{N}$-acetyl lysine and nicotinamide (to inhibit the E. coli deacetylase CobB) and purified to homogeneity (Fig. 4A). In all cases, we obtained proteins of the expected molecular masses $(33.5 \mathrm{kDa}$ for A. thaliana and $36 \mathrm{kDa}$ for P. patens; Fig. 4A). The incorporation of AcK at the desired positions of A. thaliana and P. patens mMDH1 was confirmed by LC-MS/MS (Supporting Table S6).

We next determined the in vitro kinetic parameters of the purified recombinant non-modified mMDH1 and the single-site acetylated versions. First, we focused on the direction of the reduction of OAA, in which case $\mathrm{mMDH}$ activity is needed predominantly to produce malate for its translocation to the cytosol for redox balance (Fig. 1D). We found that acetylation at positions K325 and K329 in A. thaliana mMDH1 had no significant effects on the kinetic parameters of the enzyme. In contrast, acetylation at $\mathrm{K} 334$ decreased the turnover number $\left(k_{\mathrm{cat}}\right)$ to $64.4 \%$ of the non-modified enzyme. Acetylation at K170 decreased the turnover number to $37 \%$ while increasing the affinity for OAA 
(decreasing $K_{m}$ ) to $50 \%$ of the non-modified enzyme. The same analysis with $P$. patens mMDH1 indicated that the acetylation in K172 doubles the turnover number in comparison to the non-modified enzyme (Fig. 4B), with a corresponding increase in the catalytic efficiency $\left(k_{\text {cat }} / K_{m}=23.7 \pm 3.7 \mu \mathrm{M}^{-1}\right.$ $\left.\mathrm{s}^{-1}\right)$ compared to the non-modified enzyme $\left(K_{\mathrm{cat}} / K_{m}=14.8 \pm 2.3 \mu \mathrm{M}^{-1} \mathrm{~s}^{-1}\right)$.

We further analysed mMDH activity in its respiratory role in the TCA cycle, in which OAA is generated by oxidation of malate (Fig. 1). We found that the acetylation of all analyzed single amino acid positions in A. thaliana mMDH1 affected the enzymatic parameters. Acetylation at position K170 reduces the affinity for malate to $65 \%$ compared to the non-modified enzyme (Fig. 4B). As a consequence, $\mathrm{K} 170$ acetylation reduces the catalytic efficiency $\left(K_{\text {cat }} / K_{m}=0.27 \pm 0.05 \mu \mathrm{M}^{-1} \mathrm{~s}^{-1}\right)$ to 67.5 $\%$ of the non-modified enzyme $\left(K_{\text {cat }} / K_{m}=0.40 \pm 0.04 \mu \mathrm{M}^{-1} \mathrm{~s}^{-1}\right)$. Acetylation at positions K325, K329, and $\mathrm{K} 334$ in A. thaliana mMDH1 reduced the turnover number to $76-79 \%$ compared to the nonmodified enzyme, while increasing the affinity for malate (reduced $K_{m}$ values, Fig. 4B), by $15 \%, 24$ $\%$, and $74 \%$, respectively, compared to the non-modified enzyme (Fig. 4B). The changes in the kinetic parameters of acetylated $\mathrm{mMDH} 1$ at $\mathrm{K} 334$ result in a 3-fold enhancement of the catalytic efficiency $\left(K_{\text {cat }} / K_{m}=1.34 \pm 0.56 \mu \mathrm{M}^{-1} \mathrm{~s}^{-1}\right)$. In contrast, the catalytic efficiencies of the other two enzymatic variants bearing acetylation at $\mathrm{K} 325\left(K_{\text {cat }} / K_{m}=0.37 \pm 0.05 \mu \mathrm{M}^{-1} \mathrm{~s}^{-1}\right)$ and at $\mathrm{K} 329\left(K_{\text {cat }} / K_{m}=\right.$ $\left.0.40 \pm 0.08 \mu \mathrm{M}^{-1} \mathrm{~s}^{-1}\right)$ were similar to that of the non-modified enzyme. Acetylation of P. patens mMDH1 in K172 had no effects on the enzymatic parameters in the malate oxidation direction compared to the non-modified enzyme (Fig. 4B).

Lysine residues corresponding to K172 of MDH1 of $P$. patens were also found acetylated in three angiosperm species (Zhen et al., 2016, Jiang et al., 2018, Zhou et al., 2018). We further investigated the potential conserved regulatory role of this lysine residue in A. thaliana mMDH1. We produced the recombinant A. thaliana mMDH mutant AcK169 (corresponding to K172 in P. patens) and evaluated its kinetics parameters in both directions of the reaction. We found that, similar to P. patens, the acetylation of Arabidopsis mMDH1 in K169 had no significant effects on the kinetic parameters of the enzyme in the malate oxidation reaction. In contrast, in the direction of the reduction of OAA, acetylation at K169 decreases the affinity of Arabidopsis mMDH1 for OAA by 2.3 -fold $\left(K_{m}=39 \pm 8\right.$ $\left.\mu \mathrm{M}^{-1} ; \mathrm{p}=<0,0001\right)$, with a corresponding decrease in the catalytic efficiency $\left(K_{\text {cat }} / K_{m}=15 \pm 6 \mu \mathrm{M}^{-1} \mathrm{~s}^{-}\right.$ ${ }^{1} ; \mathrm{p}=0,0027$ ) compared to the non-modified enzyme (Fig. 4B).

\section{Mobility analysis of the acetylated proteins by native PAGE}

The introduction of a PTM can cause changes in structural features of a protein, which can impact the kinetic properties. To analyse if the general organization of the non-modified enzymes is maintained in the single acetylated $\mathrm{mMDH}$ recombinant variants produced, we compared the mobility of the 
enzymes in native gel electrophoresis. The isolated proteins were run side-by-side on the same gel and analyzed for MDH activity.

We found that in homogeneous native PAGE, the recombinant mMDH1 of A. thaliana and P. patens are present as single bands very likely corresponding to dimers (Fig. 4D). A. thaliana and P. patens mMDH1 proteins differ slightly in their mobility, which is likely due to the differences in the molecular masses of the subunits (Fig. 4A) and of different gross shapes of the proteins that can be adopted in solution. The native PAGE results demonstrate that all acetylated mMDH1 versions of $A$. thaliana and $P$. patens conserved the same oligomeric arrangements observed in the non-modified proteins (Fig. 4C), indicating that the detected activity changes cannot be accounted for by changes in homomerisation.

\section{Mapping of acetylation sites on the crystal structures of MDH}

We used the crystal structures of $E$. coli $\mathrm{MDH}$ (PDB ID:1EMD) and human MDH2 (PDB ID: 2DFD) to perform homology modelling of the A. thaliana and $P$. patens structures and to map the acetylated lysine residues analysed in this study onto the molecular structures. Models of the A. thaliana and $P$. patens $\mathrm{mMDH} 1$ monomers, dimers, and tetramers show that the acetylated lysine residues are neither located in the direct vicinity of the active site (Fig. 5A-B) nor of the dimer or tetramer interfaces predicted by the constructed models (Fig. 5C-F).

Superposition analysis of the monomeric structures indicate that A. thaliana K170, P. patens K172, and E. coli $\mathrm{K} 140$ are located in the same region of the three-dimensional structure of the proteins (Fig. 6A-C), suggesting conservation of the regulatory function of these lysines by acetylation. The specific spatial orientation of the lysine side chains may differ from the orientations observed in the structures of A. thaliana and P. patens, however, since the shown structures are modelled based on the crystal structures of E. coli and human MDH.

We also observed that $A$. thaliana mMDH1 K329 and K334, two lysine positions that are conserved in plants, are located in the same amino-terminal alpha-helix as K300 and K301 of E. coli MDH (Fig. 6B, D), with $\mathrm{K} 329$ of A. thaliana mMDH1 occupying the same special position as K300 of E. coli MDH (Fig. 6B, D). While the effects of acetylation of K300 and K301 on the activity of E. coli MDH have not yet been investigated, the conserved acetylation of K300 within plants suggests a possible regulatory function. The superposition of the structures indicates that all acetylated lysine residues of A. thaliana and $P$. patens $\mathrm{mMDH} 1$ are located in different regions of the protein surface than those of human mMDH2 (Fig. 6E), anticipating different regulation strategies in plants and mammals.

\section{Discussion}

Lysine acetylation in mitochondrial proteins of evolutionarily distant species 
Acetylation of lysine residues is a particularly abundant posttranslational modification of mitochondrial and plastid proteomes (Lombard et al., 2007, König et al., 2014a, Smith-Hammond et al., 2014, Hartl et al., 2017). We detected 324 lysine acetylation sites in organellar proteins of $P$. patens gametophores. Since lysine acetylation is a highly dynamic modification and its occurrence depends on the metabolic status of the organism (Meyer et al., 2018), the acetylation pattern may differ across developmental stages, organs, and growth conditions. To reduce the influence of such intra-organismic variation, we aimed at considering analogous tissues for the comparison between $A$. thaliana and $P$. patens. Mitochondrial proteins were particularly overrepresented amongst lysine acetylated proteins detected in $P$. patens, even more so than in recent whole tissue acetylome studies of A. thaliana (Hartl et al. 2017, Uhrig et al. 2019). The number of 82 mitochondrial proteins identified here from whole $P$. patens gametophores is overall in line with 120 lysine-acetylated proteins that were detected in isolated A. thaliana mitochondria of seedlings (König et al., 2014a).

Due to the lack of evidence for a mitochondrial acetyltransferase in plants, it appears likely that mitochondrial protein acetylation mostly occurs non-enzymatically between acetyl-CoA and specific lysine residues of particularly high reactivity. The functional steady-state lysine acetylation pattern is likely set by enzymatically controlled de-acetylation, as carried out by sirtuin proteins (Lombard et al., 2007, König et al., 2014b, Anderson et al., 2017). In general, lysine acetylation removes the positive charge from the lysine side chains of proteins, increasing their hydrophobicity. However, similar to phosphorylation, lysine acetylation can affect protein function in various ways, and it can cause activation as well as inhibition of enzymes depending on the function of the particular lysine residue within the protein structure (Hosp et al., 2017).

Although the same mitochondrial proteins are frequently found acetylated in different angiosperm species, the location of the particular acetylated lysines within the protein often differs (Finkemeier et al., 2011, Hosp et al., 2017). Our study shows that this is true even across land plant phylogeny. We detected the same mitochondrial proteins acetylated in the distant land plant species $P$. patens and $A$. thaliana (in total 22 orthologous protein groups were identified) and found that the majority (66\%) of lysine positions that were acetylated in mitochondrial proteins of A. thaliana or P. patens was conserved in the orthologs of the other species as well (Supporting Table S4). However, even in the TCA cycle or PDC proteins, which showed high acetylation in both P. patens and A. thaliana (up to 4 acetylated lysines), only a low number of acetylation sites were shared (in total two) between the orthologs of the two species. A reason for the limited number of shared acetylated lysine residues detected might be the pronounced dynamics in lysine (de-) acetylation, such that each experimental condition may only detect a subset of physiologically relevant acetylations. The enzymes of the TCA cycle are known to be regulated by reversible acetylation in bacterial and mammalian cells, in dependance on the nutritional status as well as on the circadian clock (Wang et al., 2010, Masri et al., 2013, Meyer et al., 2018). Whereas caloric restrictions generally lead to deacetylation, nutrition with 
sugars leads to a strong increase in mitochondrial acetylation of all TCA cycle enzymes (Meyer et al., 2018).

Lysine residues corresponding to K170 in A. thaliana mMDH1, for which acetylation led to changes in the enzyme catalytic parameters, were found acetylated in three other species and also under different conditions. The same was observed for site K172 of MDH1 of P. patens; the corresponding lysine residues were also found acetylated in three angiosperm species. The detection of homologous acetylated lysines in distant species highlights those PTMs most likely to play a physiologically relevant role in metabolic regulation.

\section{Acetylation of lysines within a conserved hotspot modulates plant MDH activity}

In addition to the identification of acetylation of E. coli MDH and human mMDH2 (Venkat et al., 2017), recent proteomic studies report acetylation of plant mMDH proteins, albeit in different sequence positions (Supporting Table S5). To understand the potential functional significance of mMDH acetylation, we examined the enzyme characteristics in both directions of the reversible enzymatic reaction.

Our analysis of the kinetic properties of plant mMDH1 in the OAA reduction direction indicated that lysine acetylation of K172 in P. patens, and K169 (corresponding to K172 in P. patens) and K170 in A. thaliana have species-dependent opposite effects on the activities of the enzyme. Acetylation at K172 of $P$. patens mMDH1 enables the enzyme to convert OAA into pyruvate with a doubled catalytic rate compared with the non-modified protein. In contrast, the incorporation of an acetyl group at K169 or K170 in A. thaliana mMDH1 gives rise to less efficient enzymes; acetylation of K169 decreases the affinity for OAA by 2 -fold without modifying the catalytic rate, while acetylation of K170 decreases the catalytic activity by just above a third of the rate compared with the non-modified protein. A recent analysis of the protein composition of an individual average plant mitochondrion from cultured heterotrophic A. thaliana cells indicated that mMDH1 is highly abundant, with about 13.190 copies per mitochondrion (Fuchs et al., 2020). Adding the in vitro activity changes measured here to this model, the non-modified mMDH1would have the capacity to turn over 7.2 Mio molecules of OAA per second in a single mitochondrion, while acetylation at K170 would reduce this conversion capacity to 2.7 Mio molecules per second.

We found that the turnover number of $A$. thaliana non-modified mMDH1 is twice as high as that of $P$. patens non-modified mMDH1 (Fig. 4B), suggesting that the enzymes differ in their basal properties toward the substrates in the different species. These differences are likely to appear as adaptations to the metabolic environments in the species and probably result from adaptive configurations at the active site and/or of more distal regions of the proteins. Interestingly, the opposite effects of acetylation of the lysine residues in both enzymes change the turnover numbers of the acetylated proteins to values similar to those found in the non-modified enzyme of the other species analysed: the 
turnover number of $P$. patens mMDH1 acetylated in $\mathrm{K} 172$ has a similar value to that of $A$. thaliana non-modified mMDH1, while A. thaliana mMDH1 acetylated in $\mathrm{K} 170$ has a similar turnover number to that of $P$. patens non-modified mMDH1. These observations suggest that changes of the turnover number induced by acetylation of plant mMDH1 occur between minimal and maximal values that are set by the structure and catalytic mechanism of the plant enzyme. Although the precise molecular mechanism for the changes in the turnover number are still to be determined, it is interesting to note that amino acid 173 in P. patens mMDH1 (corresponding to K170 in A. thaliana mMDH1) is an arginine, which has similar properties as a permanent non-acetylated lysine (Kamieniarz and Schneider, 2009). Considering that the presence of an acetyl-lysine at position 170 of A. thaliana mMDH1 decreases the turnover number of the enzyme, the presence of an arginine in $P$. patens $\mathrm{mMDH} 1$ at the same position suggests that $P$. patens $\mathrm{mMDH} 1$ is adapted to avoid a further reduction of its enzymatic activity, at least by acetylation. This interpretation is in accordance with the lower $k_{\text {cat }}$ value we measured for the $P$. patens non-modified mMDH1 in comparison to that of the A. thaliana wild-type enzyme (Fig. 4B).

The analysis of the influences of acetylation of K172 in P.patens, and K169 and K170 in A. thaliana, on the kinetic properties of $\mathrm{mMDH} 1$ in the malate oxidation direction indicated a major difference between both plant species. While the kinetic properties of $P$. patens and A. thaliana mMDH1 acetylated at the corresponding lysine residues (K172 in P. patens and K169 in A. thaliana) were not modified, acetylation of $A$. thaliana mMDH1 at K170 reduces the enzymatic catalytic efficiency to $67.5 \%$ of the non-modified enzyme due to a high reduction of the affinity for malate (Fig. 4B).

The lysine residues corresponding to K169 and K170 of A. thaliana mMDH1 that influence the enzymatic activity of $A$. thaliana and $P$. patens mMDH1 in an acetylation-dependent manner are conserved in plants and were found to be acetylated in several species. In no case the residues were found acetylated simultaneously. The fact that K169 and K170 were found to be independently acetylated in different organs (leaves and embryos) of rice may indicate the need of fine-tuning the enzyme activity to cope with specific metabolic necessities of the organs.

Our analysis of the modelled protein structures indicates that the critical lysine residues are not near the catalytic site or in the dimer or tetramer interfaces. While the precise molecular mechanism of how acetylation influences the catalytic properties of the enzyme remains to be resolved, it appears likely that acetylation induces a conformational change that reaches across the protein structure.

\section{Acetylation of a conserved lysine at the carboxyl terminal end of $A$. thaliana mMDH1 favors the malate oxidation activity}

The C-terminus of A. thaliana mMDH1 contains three lysines (K325, K329, and K334) that were found acetylated in seedlings harvested at the beginning of the light period (König, 2014). K334 is conserved in all plant species investigated in this study, while K325 and K329 are shared by $80 \%$ and 
$\sim 70 \%$ of the investigated plant species, respectively (Fig. 3). The conservation of these lysine residues in plants suggests a possible conservation of their regulatory roles; yet to date, these residues were only found acetylated in mMDH orthologs of a few species beyond A. thaliana (Fig. 3, Supporting Table S1). We found that acetylation at the single positions K325 or K329 slightly influence the catalytic behavior of mMDH1. In contrast, acetylation at the highly conserved K334 has a major impact on the malate oxidative activity: compared to the non-modified enzyme, it decreases the catalytic efficiency of OAA reduction to $40 \%$ while increasing the catalytic efficiency of malate oxidation by 3.4-fold. A similar increase of the catalytic efficiency of the malate oxidation reaction was reported as a consequence of acetylation of $\mathrm{MDH}$ in other organisms. Acetylation of E. coli $\mathrm{MDH}$ at K140, which is located next to A. thaliana K170 and P. patens K172 (Fig. 6C), was shown to increase the catalytic efficiency of malate oxidation by 3.4-fold (Venkat et al., 2017). In the same reaction direction, the catalytic efficiency of the E. coli enzyme is also enhanced by acetylation of K99 (by 2.9-fold), while that of human mMDH2 is enhanced by acetylation of K307 (by 3.1-fold) (Venkat et al., 2017). However, different to Arabidopsis, the changes in the catalytic efficiency observed in $E$. coli and human MDH were due to increases of the enzymatic activity, while the affinity for malate was unchanged. This indicates that the activation of $\mathrm{mMDH}$ in different organisms involves alternative molecular mechanisms.

\section{Potential consequences of mMDH lysine acetylation for plant metabolism}

Our results indicate that acetylation of $A$. thaliana $\mathrm{mMDH} 1$ at the single lysine position $\mathrm{K} 170$ reduces the efficiency of the reaction in both directions by a similar value (67\% efficiency in the OAA reduction and $72 \%$ efficiency in the malate oxidation direction). Moreover, we found that acetylation at K334 favors the malate oxidation activity of A. thaliana mMDH1, as it decreases the efficiency of OAA reduction while increasing the efficiency of malate oxidation. Since acetylation of K170 and K334 allow adjustments in both directions of the reaction catalysed by mMDH1, which are fully reversible and do not require protein synthesis and/or turnover, they likely represent effective mechanisms to modulate flux through central carbon metabolism. Knowing the stoichiometry of acetylation could allow a more precise description of the impact of acetylation of a protein in metabolism (Weinert et al., 2014), even though low PTM site stoichiometry does not indicate a minor or lack of function (Xia et al., 2012, Bovdilova et al., 2019, Hansen et al., 2019). The degree of acetylation of the individual lysines analysed here is likely to vary in vivo depending on conditions and importantly on the cellular context. While PTM stoichiometry in a total tissue extract is a poor predictor of in vivo significance, the finding that acetylation of the same conserved lysine residues occurs in different plant species, even under different conditions, is an indicator of the functional relevance of the sites. 
The results of the kinetic analysis of the individual acetylated sites may inform hypotheses about possible metabolic contexts in which the analysed acetylation sites of plant mMDH may be relevant. Under conditions of high $\mathrm{NAD}^{+} / \mathrm{NADH}$ ratio in the matrix, the activity of mMDH1 in the OAA reduction direction needs to be limited, while that of the malate oxidation boosted to avoid further removal of reductant from the matrix and constraints on mitochondrial ATP production in turn (Fig. 1). Acetylation of $P$. patens $\mathrm{mMDH} 1$ at K172 increases the capacity - and potentially the rate - of OAA consumption, which will be necessary under metabolic conditions that demand a high rate of matrix $\mathrm{NAD}^{+}$provision at the expense of ATP production, e.g., during photorespiratory conditions (Fig. 1). In E. coli, the activity of MDH in the malate oxidation direction and the acetylation grade of the enzyme increase with increasing glucose concentrations (Venkat et al., 2017), suggesting an activation of the enzyme to provide sufficient capacity for enhanced flux through the TCA-cycle in its circular mode. Interestingly, bacteria grown on citrate, which favors the OAA reduction reaction of MDH, showed lower acetylation of MDH compared to growth on glucose (Wang et al., 2010, Mall et al., 2018).

The lysine residues found to be acetylated in mMDH1 of A. thaliana and P. patens most likely represent only a small proportion of the mMDH1 lysine sites that can be acetylated in vivo. As PTMs can be strictly dependent on exact conditions, other mMDH lysine residues may also be acetylated under different growth conditions, at different times of the day, or in other tissues. Additional, systematic analyses will be required in the future for their identification. Nevertheless, as in the case of other PTMs, not all acetylated lysines necessarily modify the kinetic parameters of an enzyme, as we here observed in some cases (Fig. 4B and C). Alternatively, these modifications may influence interactions with other proteins or factors that are not present in the in vitro setting devised here. Indeed, in vivo interactions between several mitochondrial proteins that were found acetylated in this study were recently reported in A. thaliana in vivo (Zhang et al., 2018), raising the possibility of further regulatory functions, e.g., by modulating metabolic channeling (Zhang et al., 2017, Zhang et al., 2018).

Our present analysis offers intriguing insights into the functional impact of single-site modifications on the enzymatic kinetics. It further provides evidence for the degrees of potential in vivo regulation, which are likely key for the regulation of cellular metabolism but are not usually accounted for by standard reductionist in vitro analyses. Acetylation of evolutionary conserved lysines provides a mechanism for tuning enzyme function to specific metabolic requirements. In vivo, the exact acetylation pattern across different lysine residues, which may dynamically change with the metabolic status of the cells, will govern the dynamic regulation of $\mathrm{mMDH}$ performance. Ideally, novel highly sensitive mass spectrometry-based methods will become available in the future, which will enable us to assess the PTM site occupancies on proteins in single cells and organelles of certain plant tissues. Such analyses would allow the direct identification of relevant PTMs that regulate metabolic activity in vivo. Also, the interplay of the different modifications involved in the modulation of $\mathrm{mMDH}$ as well 
as the conditions that lead to the specific changes in mMDH acetylation status of evolutionary conserved lysines remain to be investigated in the future.

\section{Experimental procedures}

\section{Physcomitrium patens cultivation conditions}

P. patens (Hedw.) Bruch \& Schimp. strain Gransden (Rensing et al., 2008) gametophores were cultivated on modified Knop medium plates $\left(\mathrm{KH}_{2} \mathrm{PO}_{4}\left(250 \mathrm{mg} \mathrm{L}^{-1}\right), \mathrm{KCl}\left(250 \mathrm{mg} \mathrm{L}^{-1}\right), \mathrm{MgSO}_{4} \times 7 \mathrm{H}_{2} 0\right.$ $\left(250 \mathrm{mg} \mathrm{L}^{-1}\right), \mathrm{Ca}\left(\mathrm{NO}_{3}\right)_{2} \times 4 \mathrm{H}_{2} \mathrm{O}\left(1000 \mathrm{mg} \mathrm{L}^{-1}\right), \mathrm{FeSO}_{4} \times 7 \mathrm{H}_{2} \mathrm{O}\left(12.5 \mathrm{mg} \mathrm{L}^{-1}\right), \mathrm{CuSO}_{4}(0.22 \mu \mathrm{M})$, $\mathrm{ZnSO}_{4}(0.19 \mu \mathrm{M}), \mathrm{H}_{3} \mathrm{BO}_{3}(10 \mu \mathrm{M}), \mathrm{Na}_{2} \mathrm{MoO}_{4}(0.1 \mu \mathrm{M}), \mathrm{MnCl}_{2}(2 \mu \mathrm{M}), \mathrm{CoCl}_{2}(0.23 \mu \mathrm{M})$ and $\mathrm{KI}(0.17$ $\mu \mathrm{M}), \mathrm{pH}=5.8,1 \%[\mathrm{w} / \mathrm{v}]$ agar; (Rudinger et al., 2011)) at $2^{\circ} \mathrm{C}$ under long day conditions with $16 \mathrm{~h}$ light and $8 \mathrm{~h}$ darkness at a light intensity of $65 \mu \mathrm{mol} \mathrm{m} \mathrm{m}^{-2} \mathrm{~s}^{-1}$ using neon tubes Osram HO 39W/865 Lumilux Cool Daylight. Single gametophores were transferred onto fresh plates three weeks prior to harvest and were further cultivated at $10 \mathrm{~h}$ light and $14 \mathrm{~h}$ darkness.

\section{Trypsin digestion, immuno-enrichment of lysine-acetylated peptides and LC-MS/MS data acquisition}

The lysine acetylome analysis was performed as described in detail in Lassowskat et al. (2017). Briefly, proteins were extracted from three independent replicates (including 5-6 gametophores/plate and 9-15 plates per replicate). Protein extracts were alkylated and digested with MS-grade trypsin. Peptides were desalted and enriched for lysine-acetylated proteins by immuno-purification. Total peptide samples and the immuno-enriched fractions were then analyzed via LC-MS/MS using an EASY-nLC 1200 (Thermo Fisher) coupled to a Q Exactive HF mass spectrometer (Thermo Fisher). Peptides were separated on $20 \mathrm{~cm}$ frit-less silica emitters (New Objective, $0.75 \mu \mathrm{m}$ inner diameter), packed in-house with reversed-phase ReproSil-Pur C18 AQ $1.9 \mu \mathrm{m}$ resin (Dr. Maisch). The column was kept at $50^{\circ} \mathrm{C}$ in a column oven throughout the run. The following parameters were used for acetylome analysis: Peptides were eluted for 78 min using a segmented linear gradient of 0 to $98 \%$ solvent B (solvent A $0 \% \mathrm{ACN}, 0.1 \% \mathrm{FA}$; solvent B $80 \% \mathrm{ACN}, 0.1 \% \mathrm{FA}$ ) at a flow-rate of 250 $\mathrm{nL} /$ min. Mass spectra were acquired in data-dependent acquisition mode with a Top12 method. MS spectra were acquired in the Orbitrap analyzer with a mass range of 300-1759 m/z at a resolution of 120000 FWHM, maximum IT of $55 \mathrm{~ms}$ and a target value of $3 \times 10^{6}$ ions. Precursors were selected with an isolation window of $1.2 \mathrm{~m} / \mathrm{z}$. HCD fragmentation was performed at a normalized collision energy of 25. MS/MS spectra were acquired with a target value of $5 \times 10^{4}$ ions at a resolution of $15000 \mathrm{FWHM}$, maximum IT of $150 \mathrm{~ms}$ and a fixed first mass of $\mathrm{m} / \mathrm{z} 100$. Peptides with a charge of +1 , greater than 6 , or with unassigned charge state were excluded from fragmentation for MS2, and dynamic exclusion for $30 \mathrm{~s}$ prevented repeated selection of precursors. 


\section{MS data analysis}

Raw data were processed using the MaxQuant software V1.6.17 (http://www.maxquant.org/) (Cox and Mann, 2008). MS/MS spectra were searched with the Andromeda engine against the cosmoss_V3.3 database (February 15, 2017, https://www.cosmoss.org/physcome_project /wiki/Downloads). Sequences of 248 common contaminant proteins and decoy sequences were automatically added during the search. Trypsin specificity was required and a maximum of four missed cleavages were allowed. Minimal peptide length was set to seven amino acids. Carbamidomethylation of cysteine residues was set as fixed, oxidation of methionine and protein N-terminal acetylation, acetylation of lysines as variable modifications. Light and medium dimethylation of lysines and peptide $\mathrm{N}$-termini were set as labels. Peptide-spectrum-matches and proteins were retained if they were below a false discovery rate of $1 \%$, modified peptides were additionally filtered for a score $\geq 35$ and a delta score of $\geq 6$ to remove low quality identifications. Match between runs and requantify options were enabled. For acetylome analyses reverse hits and contaminants were removed. Acetylation sites were filtered for a localization probability of $\geq 0.75$.

\section{MS analyses of the recombinant mMDH proteins}

The recombinant proteins (10 $\mu \mathrm{g}$ of each) were alkylated and trypsin-digested in urea buffer as described previously (König et al., 2014b). Up to $500 \mathrm{ng}$ of the trypsinated peptides were analysed by LC-MS/MS as described above with the following differences: Mass spectra were acquired in datadependent acquisition mode with a Top15 method. MS spectra were acquired in the Orbitrap analyzer with a mass range of $300-1759 \mathrm{~m} / \mathrm{z}$ at a resolution of $60000 \mathrm{FWHM}$, maximum IT of $30 \mathrm{~ms}$ and a target value of $3 \times 10^{6}$ ions. Precursors were selected with an isolation window of $1.3 \mathrm{~m} / \mathrm{z}$. MS/MS spectra were acquired with a target value of $1 \times 10^{5}$ ions at a resolution of $15000 \mathrm{FWHM}$, maximum IT of $55 \mathrm{~ms}$. For the data analysis, MaxQuant software was used with standard settings and the following additions: MS/MS spectra were searched with the Andromeda engine against the Uniprot $E$. coli 12 database including either the protein sequences of $P$. patens or A. thaliana mMDH. Trypsin specificity was required and a maximum of two missed cleavages were allowed. Carbamidomethylation of cysteine residues was set as fixed, oxidation of methionine and acetylation of lysines as variable modifications. Results of all $\mathrm{mMDH}$ peptides from modification specific peptides table are presented in Supporting Table S6. It has to be noted that some acetylation sites are present on the recombinant $\mathrm{mMDH}$ proteins due to untargeted acetylation from $E$. coli, however these acetylation sites are only present at very low level (mostly below 1\%) as estimated from the peptide intensities compared to the unmodified peptide intensities. 


\section{Functional classification and prediction of subcellular localization}

Proteins were functional annotated based on the P. patens V3.3 database. Localization was inferred from the experimental dataset by Müller et al. (2014) and the GO_CC annotation provided in Lang et al. (2018).

\section{Comparison of acetylated lysines of putative mitochondrial orthologs}

Sequences of the 120 acetylated A. thaliana proteins identified in König et al. (2014a) were obtained from Tair (https://www.arabidopsis.org/tools/bulk/sequences/index.jsp) and sequences of those $P$. patens proteins that were acetylated and classified were obtained from Peatmoss (https://peatmoss.online.uni-marburg.de/ppatens_db/pp_search_input.php) (Fernandez-Pozo et al., 2020). Amino acid sequences of both species were combined and clustered using the multiple alignment program MAFFT Version 7 with default settings (Katoh et al., 2002, Katoh et al., 2019). P. patens and A. thaliana proteins sequences of orthologous groups were aligned again with Muscle (Edgar, 2004) using the Mega 7 software (Kumar et al., 2016). In total 22 clusters of P. patens and A. thaliana proteins were identified with homologs sharing sequence similarity higher than $40 \%$. In each cluster different numbers of paralogous proteins of each species were included. Acetylation sites and amino acid positions of proteins of each orthologous group were compared. Acetylation sites were listed and positions extracted from the original alignment (Table 1, Supporting Table S4, Supporting Data S1).

\section{Phylogenetic analysis, comparison of sequence conservation and lysine acetylation patterns of mMDHs of different plant species}

Published acetylome datasets were inspected for the presence of the mitochondrial malate dehydrogenase. In total 17 datasets for 10 different flowering plants were included in the analysis (Supporting Table S5). For seven flowering plant species lysine acetylation of mMDH proteins was detected. Plant tissues or organs, in which these lysine acetylated mMDHs were detected, are given in Supporting Table S5. Putative mMDH orthologs of these species were identified by Basic Local Alignment Tool (BLAST) searches using the A. thaliana $\mathrm{mMDH} 1$ protein sequence as query against nonredundant protein database (BLASTP) and against translated nucleotide database (TBLASTN) (Altschul et al., 1990) of different sources. Sequence data of the selected angiosperm species $C$. sinensis, V. vinifera, $O$. sativa, B. distachyon, $F$. ananass and the green algae $C$. reinhardii were deposited at NCBI (www.ncbi.nlm.nih.gov) and sequence data of $P$. patens and M. polymorpha are deposited at Phytozome v12.0 (https://phytozome.jgi.doe.gov/pz/portal.html). For putative mMDH orthologs of Azolla and Salvinia, sequences are available in the Fernbase (www.fernbase.org). For $P$. sativum the URGI database was used as sequence source (Kreplak et al., 2019). The Anthoceros 
sequences were retrieved from the recently published A. agrestis, Bonn v1 strain genome v1.1 (Li et al., 2020). Best 2-10 hits were downloaded for each species and were aligned in the MEGA alignment explorer with the MUSCLE tool (Tamura et al., 2015, Kumar et al., 2016) followed by manual adjustment.

Amino acid sequence alignment was used to calculate a Neighbor joining phylogeny with 1000 bootstraps (Poisson model, gamma distributed, partial deletion with cut off 90\%) including the peroxisomal (AT2G22780), plastidial (AT3G47520) and mitochondrial A. thaliana mMDH proteins. With a reduced dataset (Supporting Data S2) including all MDH proteins of the identified mitochondrial cluster, glyoxysomal/peroxisomal and plastid MDHs of $P$. patens and A. thaliana and the MDH of $E$. coli and the $\mathrm{mMDH} 2$ of human, we constructed a Maximum Likelihood phylogram using the IQ-tree software version 1.6.12 (Trifinopoulos et al., 2016). We applied the LG+G4 model for sequence evolution as the best fitting model identified by ModelFinder (Kalyaanamoorthy et al., 2017). We determined the confidence of each node from 1,000 bootstrap replicates with ultrafast bootstrap approximation "UFBoot" (Hoang et al., 2018). In parallel we calculated a Neighbor joining phylogeny with same settings as described above (Supporting Fig. S1). Sequences of mMDH proteins identified to be acetylated were aligned and conservation was displayed using the GeneDoc software (https://genedoc.software.informer.com/2.7/).

\section{Cloning of A. thaliana mMDH1 into the expression vector}

The cDNA encoding the mMDH1 mature protein (without signal peptide) from $A$. thaliana (Hüdig et al., 2015) was introduced in pCDF PylT-1 vector (SmR; $T 7$ promoter; $T 7$ terminator) (Neumann et al., 2009). This vector allows the expression of proteins fused to an N-terminal His tag for purification by Ni-NTA affinity chromatography. The mMDH cassette was obtained by PCR using primers mmdh1_GibFw and mmdh1_GibRv and cloned by Gibson assembly. The sequence of the resulting plasmid was verified by sequencing. The pCDFmdh1 vector was used as template for site directed mutagenesis by PCR, to change specific lysine codons to the amber codon, using the primers listed in Supporting Table S6. The following constructs were generated: pCDFmdh1amb170, pCDFmdh1amb325, pCDFmdh1amb329, pCDFmdh1amb334. The incorporation of the desired nucleotide changes was confirmed by sequencing (Macrogen).

\section{Cloning of $P$. patens mMDH1 into the expression vector}

The coding sequence of mMDH1 (without signal peptide) of $P$. patens Gransden (gene Pp1s201_6V6.1) (Lang et al., 2018) was amplified with primers MfeI_PpMDH1_f and KpnI_PpMDH1_r (Supporting Table S6). The mMDH1 version with the amber codon for amino acid position 172 was generated by overlap extension PCR with internal primers (PpMDH1_KStop_f and 
PpMDH1_KStop_r, Supporting Table S6). The fragments were introduced into the pCDF PylT-1 vector (SmR; $T 7$ promoter; $T 7$ terminator) between restriction sites MfeI and KpnI (SmR; T7 promoter; $T 7$ terminator) (Neumann et al., 2009) to generate N-terminally His-tagged mMDH1. The incorporation of the desired nucleotide changes was confirmed by sequencing (Macrogen).

\section{Expression and purification of acetylated mMDHs}

All pCDF PylT expression constructs carrying the mMDH1 versions with an amber codon instead of the codon for the lysine to be acetylated and those carrying the corresponding wild-type mMDH1 were transformed into E. coli Rosetta2 (DE3). Plasmid pBK-AcKRS3, carrying the acetyl-lysyl-tRNA synthetase (AcKRS) (Neumann et al., 2008), was co-transformed with the pCDF PylT plasmids. For heterologous protein production, transformed cells were grown in $400 \mathrm{ml} \mathrm{LB}$ medium in the presence of the appropriate antibiotic in each case at $37^{\circ} \mathrm{C}$ and $16 \mathrm{~g}$ to an OD600 of 0.4 - 0.6. The growth medium was supplemented with $10 \mathrm{mM} \mathrm{N}$ '-acetyl-lysine and $20 \mathrm{mM}$ nicotinamide $30 \mathrm{~min}$ before induction. To induce the expression of the heterologous protein, $1 \mathrm{mM} \mathrm{IPTG}$ was added to the culture and the cells were incubated for $20 \mathrm{~h}$ at $30^{\circ} \mathrm{C}$ and $16 \mathrm{~g}$. The cellular culture was harvested at $6,000 \mathrm{~g}$ for $15 \mathrm{~min}$, and pellets were stored at $-20^{\circ} \mathrm{C}$ until use.

For protein extraction, pellets were thawed on ice, resuspended in $20 \mathrm{mM}$ Tris- $\mathrm{HCl}(\mathrm{pH} 8.0)$ containing $500 \mathrm{mM} \mathrm{NaCl}, 5 \mathrm{mM}$ imidazole, $1 \mathrm{mM}$ phenylmethylsulfonyl fluoride and a spatula-tip of lysozyme, sonicated and centrifuged at $14,000 \mathrm{~g}$ for $20 \mathrm{~min}$ at $4^{\circ} \mathrm{C}$. The supernatant was used for protein purification by gravity-flow immobilized metal ion chromatography on nickel-nitrilotriacetic acid agarose (Ni-NTA Agarose, ThermoFisher). The column was pre-equilibrated with $20 \mathrm{mM}$ Tris$\mathrm{HCl}$ buffer containing $500 \mathrm{mM} \mathrm{NaCl}$ and $5 \mathrm{mM}$ imidazole. After loading of the supernatant, the columns were washed in four steps with $500 \mathrm{mM} \mathrm{NaCl}$ in $20 \mathrm{mM}$ Tris- $\mathrm{HCl}$ (pH 8.0) containing increasing concentrations of imidazole $(5,40$ and $60 \mathrm{mM})$. Protein elution was performed in four steps of $500 \mu \mathrm{l}$ of $20 \mathrm{mM}$ Tris- $\mathrm{HCl}, 500 \mathrm{mM} \mathrm{NaCl}$ and $200 \mathrm{mM}$ imidazole. Finally, Vivaspin ${ }^{\circledR} 10 \mathrm{~K}$ columns (Satorius, Germany) were used for protein concentration and buffer exchanging (HEPES 50 $\mathrm{mM}, \mathrm{pH}$ 7.4). The isolated recombinant proteins were analysed by SDS-PAGE and LC-MS/MS (see above) to verify integrity and purity. Protein concentration was determined by Amido black assay (Dieckmann-Schuppert and Schnittler, 1997).

\section{Kinetic characterization of acetylated mMDH1}

$\mathrm{MDH}$ activity assays were carried on in a Synergy HT Biotek Plate Reader system at $25^{\circ} \mathrm{C}$. OAA reduction was determined following the oxidation of NADH at $340 \mathrm{~nm}\left(\varepsilon=6.2 \mathrm{~cm}^{-1} \mathrm{mM}^{-1}\right)$. The assay medium contained $50 \mathrm{mM}$ Hepes- $\mathrm{KOH} \mathrm{pH} 7.5,10 \mathrm{mM} \mathrm{MgCl}_{2}, 5 \mathrm{mM} \mathrm{NADH}$ and variable concentrations of $\mathrm{OAA}$ in the range 0.01 to $4 \mathrm{mM}$. Malate oxidation was determined following the 
reduction of NAD at $340 \mathrm{~nm}$ in an assay medium containing $50 \mathrm{mM}$ Hepes- $\mathrm{KOH} \mathrm{pH} \mathrm{7.5,} 10 \mathrm{mM}$ $\mathrm{MgCl}_{2,4} \mathrm{mM} \mathrm{NAD}$ and a variable concentration of malate in the range 0.01 to $10 \mathrm{mM}$.

The reactions were started by the addition of the substrate. The kinetic constants were calculated with a minimum of three different enzyme batches each in at least triplicate determinations and adjusted to Michaelis-Menten equation by non-linear regression with Prism 6 (GraphPad Software).

\section{Gel electrophoresis}

SDS-PAGE was performed using 14\% (w/v) polyacrylamide gels according to (Laemmli, 1970) and the molecular weight markers Spectra Multicolor Broad Range Protein Ladder from ThermoFisher Scientific. Proteins were visualized by staining with Coomassie Brilliant Blue. Purified enzymes were analyzed on a non-denaturing $7 \%(\mathrm{w} / \mathrm{v})$ polyacrylamide gel using the markers $\beta$-amlyase (200 kDa), alcohol dehydrogenase $(150 \mathrm{kDa})$ and bovine serum albumin $(66 \mathrm{kDa})$ from Sigma Aldrich. In-gel mMDH activity assays were performed by incubating the gels in the dark at room temperature in a reaction buffer containing $50 \mathrm{mM} \mathrm{K}_{2} \mathrm{PO}_{4}(\mathrm{pH} 7.4), 5 \mathrm{mM}$ malate, $0.2 \mathrm{mM} \mathrm{NAD}, 0.05 \%(\mathrm{w} / \mathrm{v}) \mathrm{NBT}$ and $10 \mu \mathrm{M}$ PMS as described in Hüdig et al. (2015).

\section{Statistical analyses}

Statistical analyses of the kinetic data were performed on data from at least three biological replicates, each measured at least in triplicate. To verify the statistical differences, $\mathrm{P}$ values were determined using unpaired t-test. Statistical analyses of the distribution of acetylation in organellar proteins and non-organellar proteins were performed with chi-square test using Excel package, XLSTAT (Addinsoft).

\section{Homology modelling of mMDH 3-D structures}

The 3-D models of E. coli MDH (PDB code 1EMD) and H. sapiens MDH2 (PDB code 2DFD) structures were obtained from the RSCB Protein Data Bank (https://www.rcsb.org/). Both A. thaliana and $P$. patens $\mathrm{mMDH} 1$ models were obtained by using the phyre2 protein structure prediction server (http://www.sbg.bio.ic.ac.uk/ phyre2/html/page.cgi?id=index) with the corresponding amino acid sequence as input. The ligand molecules $\mathrm{NAD}^{+}$and citrate were manually fitted in by aligning the structure of the $H$. sapiens MDH2 (pdb code 2DFD) to the modelled structures of A. thaliana and $P$. patens mMDH1. Images of the models were prepared using PyMOL v.2.4.0 by Schrödinger (https://pymol.org/).

\section{Data statement}


The data underlying this article are available in the article and in its online Supporting material.

\section{Author contributions}

M.B., M.M.B., A.B., M.H., and L.R. performed biochemical studies on recombinant proteins. M.E. and J.G. generated the lysine acetylome of P. patens. M.S., I.F, M.S-R, and V.G.M. designed the experiments and analyzed the data together with other researchers; I.F, M.S-R, and V.G.M. conceived the project. I.F, M.S-R, and V.G.M. wrote the article with contributions of M.S., M.B. and M.E.; V.G.M. agrees to serve as the author responsible for contact and ensures communication.

\section{Acknowledgements}

This work was enabled through the collaborative DFG research grant PAK918 (IF1655/3-1, MA2379/14-1, SCHA1953/3-1, and SCHW1719/5-1) as part of the 'Plant Mitochondria in New Light' initiative and INST 211/744-1 FUGG for LC-MS/MS analyses. Work of MBB in the group of VGM was funded by the StayConnected-Grant of the Heinrich Heine University Düsseldorf. The pBK PylS and pCDF PylT vectors were provided by Dr. J. Chin (University of Cambridge) under a noncommercial material transfer agreement to IF. We thank Astrid Höppner (HHU, Düsseldorf) for her help with the structural analysis and Paulina Pieloch and Dr. Jürgen Eirich for technical assistance within the MS Proteomics Unit Biology of Plants of the University of Muenster. The co-authors have no conflict of interest to declare.

\section{Supporting material}

Supporting Data S1. Protein alignment of identified orthologous groups.

Supporting Data S2. Sequence alignment of the MDH dataset for phylogenetic construction.

Supporting Fig. S1. Mitochondrial malate dehydrogenases along land plant phylogeny.

Supporting Table S1. Proteins identified in total extracts from Physcomitrella gametophores, which were immuno-enriched for lysine-acetylated peptides.

Supporting Table S2. Lysine-acetylated proteins identified in total extracts from Physcomitrella gametophores after immuno-enrichment of lysine-acetylated peptides.

Supporting Table S3. Acetylated lysine sites

Supporting Table S4. Conservation of lysine acetylation between mitochondrial proteins of orthologous groups identified in A. thaliana and P. patens.

Supporting Table S5. Lysine acetylome data of mMDH proteins in plants, human and E. coli 
bioRxiv preprint doi: https://doi.org/10.1101/2020.10.30.362046; this version posted June 15, 2021. The copyright holder for this preprint (which was not certified by peer review) is the author/funder. All rights reserved. No reuse allowed without permission.

Supporting Table S6. Unique peptides of mMDH recombinant proteins purified from E. coli.

Supporting Table S7. Primers used in this work. 


\section{References}

Allfrey, V.G., Faulkner, R. and Mirsky, A.E. (1964) Acetylation and Methylation of Histones and Their Possible Role in the Regulation of Rna Synthesis. Proceedings of the National Academy of Sciences of the United States of America, 51, 786-794.

Altschul, S.F., Gish, W., Miller, W., Myers, E.W. and Lipman, D.J. (1990) Basic local alignment search tool. J. Mol. Biol., 215, 403-410.

Anderson, K.A., Madsen, A.S., Olsen, C.A. and Hirschey, M.D. (2017) Metabolic control by sirtuins and other enzymes that sense $\mathrm{NAD}(+), \mathrm{NADH}$, or their ratio. Biochim Biophys Acta Bioenerg, 1858, 991-998.

Bovdilova, A., Alexandre, B.M., Hoppner, A., Luis, I.M., Alvarez, C.E., Bickel, D., Gohlke, H., Decker, C., Nagel-Steger, L., Alseekh, S., Fernie, A.R., Drincovich, M.F., Abreu, I.A. and Maurino, V.G. (2019) Posttranslational Modification of the NADP-Malic Enzyme Involved in C4 Photosynthesis Modulates the Enzymatic Activity during the Day. Plant Cell, 31, 2525-2539.

Bremer, B., Bremer, K., Chase, M., Fay, M., Reveal, J., Soltis, D., Soltis, P. and Stevens, P. (2009) An update of the Angiosperm Phylogeny Group classification for the orders and families of flowering plants: APG III. Bot. J. Linn. Soc., 161, 105-121.

Choudhary, C., Weinert, B.T., Nishida, Y., Verdin, E. and Mann, M. (2014) The growing landscape of lysine acetylation links metabolism and cell signalling. Nat. Rev. Mol. Cell Biol., 15, 536-550.

Cox, J. and Mann, M. (2008) MaxQuant enables high peptide identification rates, individualized p.p.b.-range mass accuracies and proteome-wide protein quantification. Nat. Biotechnol., 26, 1367-1372.

Dieckmann-Schuppert, A. and Schnittler, H.J. (1997) A simple assay for quantification of protein in tissue sections, cell cultures, and cell homogenates, and of protein immobilized on solid surfaces. Cell Tissue Res., 288, 119-126.

Edgar, R.C. (2004) MUSCLE: multiple sequence alignment with high accuracy and high throughput. Nucleic Acids Res., 32, 1792-1797.

Fang, X., Chen, W., Zhao, Y., Ruan, S., Zhang, H., Yan, C., Jin, L., Cao, L., Zhu, J., Ma, H. and Cheng, Z. (2015) Global analysis of lysine acetylation in strawberry leaves. Front Plant Sci, 6, 739.

Fernandez-Pozo, N., Haas, F.B., Meyberg, R., Ullrich, K.K., Hiss, M., Perroud, P.F., Hanke, S., Kratz, V., Powell, A.F., Vesty, E.F., Daum, C.G., Zane, M., Lipzen, A., Sreedasyam, A., Grimwood, J., Coates, J.C., Barry, K., Schmutz, J., Mueller, L.A. and Rensing, S.A. (2020) PEATmoss (Physcomitrella Expression Atlas Tool): a unified gene expression atlas for the model plant Physcomitrella patens. Plant J., 102, 165-177.

Finkemeier, I., Laxa, M., Miguet, L., Howden, A.J. and Sweetlove, L.J. (2011) Proteins of diverse function and subcellular location are lysine acetylated in Arabidopsis. Plant Physiol., 155, 1779-1790.

Fuchs, P., Rugen, N., Carrie, C., Elsasser, M., Finkemeier, I., Giese, J., Hildebrandt, T.M., Kuhn, K., Maurino, V.G., Ruberti, C., Schallenberg-Rudinger, M., Steinbeck, J., Braun, H.P., Eubel, H., Meyer, E.H., Muller-Schussele, S.J. and Schwarzlander, M. (2020) Single organelle function and organization as estimated from Arabidopsis mitochondrial proteomics. Plant J., 101, 420-441.

Gleason, W.B., Fu, Z., Birktoft, J. and Banaszak, L. (1994) Refined crystal structure of mitochondrial malate dehydrogenase from porcine heart and the consensus structure for dicarboxylic acid oxidoreductases. Biochemistry, 33, 2078-2088.

Guan, K.L. and Xiong, Y. (2011) Regulation of intermediary metabolism by protein acetylation. Trends Biochem. Sci., 36, 108-116. 
Hanning, I. and Heldt, H.W. (1993) On the Function of Mitochondrial Metabolism during Photosynthesis in Spinach (Spinacia oleracea L.) Leaves (Partitioning between Respiration and Export of Redox Equivalents and Precursors for Nitrate Assimilation Products). Plant Physiol., 103, 1147-1154.

Hansen, B.K., Gupta, R., Baldus, L., Lyon, D., Narita, T., Lammers, M., Choudhary, C. and Weinert, B.T. (2019) Analysis of human acetylation stoichiometry defines mechanistic constraints on protein regulation. Nature communications, 10, 1055.

Hartl, M., Fussl, M., Boersema, P.J., Jost, J.O., Kramer, K., Bakirbas, A., Sindlinger, J., Plochinger, M., Leister, D., Uhrig, G., Moorhead, G.B., Cox, J., Salvucci, M.E., Schwarzer, D., Mann, M. and Finkemeier, I. (2017) Lysine acetylome profiling uncovers novel histone deacetylase substrate proteins in Arabidopsis. Mol. Syst. Biol., 13, 949.

He, D., Wang, Q., Li, M., Damaris, R.N., Yi, X., Cheng, Z. and Yang, P. (2016) Global Proteome Analyses of Lysine Acetylation and Succinylation Reveal the Widespread Involvement of both Modification in Metabolism in the Embryo of Germinating Rice Seed. Journal of proteome research, 15, 879-890.

Hirschey, M.D., Shimazu, T., Huang, J.Y., Schwer, B. and Verdin, E. (2011) SIRT3 regulates mitochondrial protein acetylation and intermediary metabolism. Cold Spring Harb Symp Quant Biol, 76, 267-277.

Hoang, D.T., Chernomor, O., von Haeseler, A., Minh, B.Q. and Vinh, L.S. (2018) UFBoot2: Improving the Ultrafast Bootstrap Approximation. Mol. Biol. Evol., 35, 518-522.

Hosp, F., Lassowskat, I., Santoro, V., De Vleesschauwer, D., Fliegner, D., Redestig, H., Mann, M., Christian, S., Hannah, M.A. and Finkemeier, I. (2017) Lysine acetylation in mitochondria: From inventory to function. Mitochondrion, 33, 58-71.

Hüdig, M., Maier, A., Scherrers, I., Seidel, L., Jansen, E.E., Mettler-Altmann, T., Engqvist, M.K. and Maurino, V.G. (2015) Plants Possess a Cyclic Mitochondrial Metabolic Pathway similar to the Mammalian Metabolic Repair Mechanism Involving Malate Dehydrogenase and 1-2-Hydroxyglutarate Dehydrogenase. Plant Cell Physiol., 56, $1820-1830$.

Imai, S., Armstrong, C.M., Kaeberlein, M. and Guarente, L. (2000) Transcriptional silencing and longevity protein Sir2 is an NAD-dependent histone deacetylase. Nature, 403, 795-800.

Inuzuka, H., Gao, D., Finley, L.W., Yang, W., Wan, L., Fukushima, H., Chin, Y.R., Zhai, B., Shaik, S., Lau, A.W., Wang, Z., Gygi, S.P., Nakayama, K., TeruyaFeldstein, J., Toker, A., Haigis, M.C., Pandolfi, P.P. and Wei, W. (2012) Acetylation-dependent regulation of Skp2 function. Cell, 150, 179-193.

Jiang, J., Gai, Z., Wang, Y., Fan, K., Sun, L., Wang, H. and Ding, Z. (2018) Comprehensive proteome analyses of lysine acetylation in tea leaves by sensing nitrogen nutrition. BMC Genomics, 19, 840.

Journet, E.P., Neuburger, M. and Douce, R. (1981) Role of Glutamate-oxaloacetate Transaminase and Malate Dehydrogenase in the Regeneration of NAD for Glycine Oxidation by Spinach leaf Mitochondria. Plant Physiol., 67, 467-469.

Kalyaanamoorthy, S., Minh, B.Q., Wong, T.K.F., von Haeseler, A. and Jermiin, L.S. (2017) ModelFinder: fast model selection for accurate phylogenetic estimates. Nat. Methods, 14, 587-589.

Kamieniarz, K. and Schneider, R. (2009) Tools to tackle protein acetylation. Chem. Biol., 16, 1027-1029.

Katoh, K., Misawa, K., Kuma, K. and Miyata, T. (2002) MAFFT: a novel method for rapid multiple sequence alignment based on fast Fourier transform. Nucleic Acids Res., 30, 3059-3066. 
Katoh, K., Rozewicki, J. and Yamada, K.D. (2019) MAFFT online service: multiple sequence alignment, interactive sequence choice and visualization. Brief Bioinform, 20, 1160-1166.

König, A.C., Hartl, M., Boersema, P.J., Mann, M. and Finkemeier, I. (2014a) The mitochondrial lysine acetylome of Arabidopsis. Mitochondrion, 19, 252-260.

König, A.C., Hartl, M., Pham, P.A., Laxa, M., Boersema, P.J., Orwat, A., Kalitventseva, I., Plochinger, M., Braun, H.P., Leister, D., Mann, M., Wachter, A., Fernie, A.R. and Finkemeier, I. (2014b) The Arabidopsis class II sirtuin is a lysine deacetylase and interacts with mitochondrial energy metabolism. Plant Physiol., 164, 1401-1414.

Kreplak, J., Madoui, M.A., Capal, P., Novak, P., Labadie, K., Aubert, G., Bayer, P.E., Gali, K.K., Syme, R.A., Main, D., Klein, A., Berard, A., Vrbova, I., Fournier, C., d'Agata, L., Belser, C., Berrabah, W., Toegelova, H., Milec, Z., Vrana, J., Lee, H., Kougbeadjo, A., Terezol, M., Huneau, C., Turo, C.J., Mohellibi, N., Neumann, P., Falque, M., Gallardo, K., McGee, R., Tar'an, B., Bendahmane, A., Aury, J.M., Batley, J., Le Paslier, M.C., Ellis, N., Warkentin, T.D., Coyne, C.J., Salse, J., Edwards, D., Lichtenzveig, J., Macas, J., Dolezel, J., Wincker, P. and Burstin, J. (2019) A reference genome for pea provides insight into legume genome evolution. Nat. Genet., 51, 1411-1422.

Kumar, S., Stecher, G. and Tamura, K. (2016) MEGA7: Molecular Evolutionary Genetics Analysis Version 7.0 for Bigger Datasets. Mol. Biol. Evol., 33, 1870-1874.

Laemmli, U.K. (1970) Cleavage of structural proteins during the assembly of the head of bacteriophage T4. Nature, 227, 680-685.

Lang, D., Ullrich, K.K., Murat, F., Fuchs, J., Jenkins, J., Haas, F.B., Piednoel, M., Gundlach, H., Van Bel, M., Meyberg, R., Vives, C., Morata, J., Symeonidi, A., Hiss, M., Muchero, W., Kamisugi, Y., Saleh, O., Blanc, G., Decker, E.L., van Gessel, N., Grimwood, J., Hayes, R.D., Graham, S.W., Gunter, L.E., McDaniel, S.F., Hoernstein, S.N.W., Larsson, A., Li, F.W., Perroud, P.F., Phillips, J., Ranjan, P., Rokshar, D.S., Rothfels, C.J., Schneider, L., Shu, S., Stevenson, D.W., Thummler, F., Tillich, M., Villarreal Aguilar, J.C., Widiez, T., Wong, G.K., Wymore, A., Zhang, Y., Zimmer, A.D., Quatrano, R.S., Mayer, K.F.X., Goodstein, D., Casacuberta, J.M., Vandepoele, K., Reski, R., Cuming, A.C., Tuskan, G.A., Maumus, F., Salse, J., Schmutz, J. and Rensing, S.A. (2018) The Physcomitrella patens chromosome-scale assembly reveals moss genome structure and evolution. Plant J., 93, 515-533.

Lassowskat, I., Hartl, M., Hosp, F., Boersema, P.J., Mann, M. and Finkemeier, I. (2017) Dimethyl-Labeling-Based Quantification of the Lysine Acetylome and Proteome of Plants. Methods in molecular biology, 1653, 65-81.

Li, F.W., Nishiyama, T., Waller, M., Frangedakis, E., Keller, J., Li, Z., Fernandez-Pozo, N., Barker, M.S., Bennett, T., Blazquez, M.A., Cheng, S., Cuming, A.C., de Vries, J., de Vries, S., Delaux, P.M., Diop, I.S., Harrison, C.J., Hauser, D., HernandezGarcia, J., Kirbis, A., Meeks, J.C., Monte, I., Mutte, S.K., Neubauer, A., Quandt, D., Robison, T., Shimamura, M., Rensing, S.A., Villarreal, J.C., Weijers, D., Wicke, S., Wong, G.K., Sakakibara, K. and Szovenyi, P. (2020) Anthoceros genomes illuminate the origin of land plants and the unique biology of hornworts. Nat Plants, 6, 259-272.

Lin, H., Su, X. and He, B. (2012) Protein lysine acylation and cysteine succination by intermediates of energy metabolism. ACS Chem Biol, 7, 947-960.

Lombard, D.B., Alt, F.W., Cheng, H.L., Bunkenborg, J., Streeper, R.S., Mostoslavsky, R., Kim, J., Yancopoulos, G., Valenzuela, D., Murphy, A., Yang, Y., Chen, Y., Hirschey, M.D., Bronson, R.T., Haigis, M., Guarente, L.P., Farese, R.V., Jr., 
Weissman, S., Verdin, E. and Schwer, B. (2007) Mammalian Sir2 homolog SIRT3 regulates global mitochondrial lysine acetylation. Mol. Cell. Biol., 27, 8807-8814.

Mall, A., Sobotta, J., Huber, C., Tschirner, C., Kowarschik, S., Bacnik, K., Mergelsberg, M., Boll, M., Hugler, M., Eisenreich, W. and Berg, I.A. (2018) Reversibility of citrate synthase allows autotrophic growth of a thermophilic bacterium. Science, $\mathbf{3 5 9}$, 563-567.

Masri, S., Patel, V.R., Eckel-Mahan, K.L., Peleg, S., Forne, I., Ladurner, A.G., Baldi, P., Imhof, A. and Sassone-Corsi, P. (2013) Circadian acetylome reveals regulation of mitochondrial metabolic pathways. Proceedings of the National Academy of Sciences of the United States of America, 110, 3339-3344.

Matsuzaki, H., Daitoku, H., Hatta, M., Aoyama, H., Yoshimochi, K. and Fukamizu, A. (2005) Acetylation of Foxo1 alters its DNA-binding ability and sensitivity to phosphorylation. Proceedings of the National Academy of Sciences of the United States of America, 102, 11278-11283.

Maurino, V.G. and Engqvist, M.K. (2015) 2-Hydroxy Acids in Plant Metabolism. The Arabidopsis book / American Society of Plant Biologists, 13, e0182.

Melo-Braga, M.N., Verano-Braga, T., Leon, I.R., Antonacci, D., Nogueira, F.C., Thelen, J.J., Larsen, M.R. and Palmisano, G. (2012) Modulation of protein phosphorylation, $\mathrm{N}$-glycosylation and Lys-acetylation in grape (Vitis vinifera) mesocarp and exocarp owing to Lobesia botrana infection. Mol. Cell. Proteomics, 11, 945-956.

Meyer, J.G., Softic, S., Basisty, N., Rardin, M.J., Verdin, E., Gibson, B.W., Ilkayeva, O., Newgard, C.B., Kahn, C.R. and Schilling, B. (2018) Temporal dynamics of liver mitochondrial protein acetylation and succinylation and metabolites due to high fat diet and/or excess glucose or fructose. PloS one, 13, e0208973.

Moller, I.M., Igamberdiev, A.U., Bykova, N.V., Finkemeier, I., Rasmusson, A.G. and Schwarzlander, M. (2020) Matrix Redox Physiology Governs the Regulation of Plant Mitochondrial Metabolism through Posttranslational Protein Modifications. Plant Cell, 32, 573-594.

Müller, S.J., Lang, D., Hoernstein, S.N., Lang, E.G., Schuessele, C., Schmidt, A., Fluck, M., Leisibach, D., Niegl, C., Zimmer, A.D., Schlosser, A. and Reski, R. (2014) Quantitative analysis of the mitochondrial and plastid proteomes of the moss Physcomitrella patens reveals protein macrocompartmentation and microcompartmentation. Plant Physiol., 164, 2081-2095.

Murphey, W.H., Kitto, G.B., Everse, J. and Kaplan, N. (1967) Malate dehydrogenases. I. A survey of molecular size measured by gel filtration. Biochemistry, 6, 603-610.

Neumann, H., Hancock, S.M., Buning, R., Routh, A., Chapman, L., Somers, J., OwenHughes, T., van Noort, J., Rhodes, D. and Chin, J.W. (2009) A method for genetically installing site-specific acetylation in recombinant histones defines the effects of H3 K56 acetylation. Mol. Cell, 36, 153-163.

Neumann, H., Peak-Chew, S.Y. and Chin, J.W. (2008) Genetically encoding N(epsilon)acetyllysine in recombinant proteins. Nat. Chem. Biol., 4, 232-234.

Noyes, B.E., Glatthaar, B.E., Garavelli, J.S. and Bradshaw, R.A. (1974) Structural and functional similarities between mitochondrial malate dehydrogenase and L-3hydroxyacyl CoA dehydrogenase. Proceedings of the National Academy of Sciences of the United States of America, 71, 1334-1338.

Qiu, Y.L., Li, L., Wang, B., Chen, Z., Knoop, V., Groth-Malonek, M., Dombrovska, O., Lee, J., Kent, L., Rest, J., Estabrook, G.F., Hendry, T.A., Taylor, D.W., Testa, C.M., Ambros, M., Crandall-Stotler, B., Duff, R.J., Stech, M., Frey, W., Quandt, D. and Davis, C.C. (2006) The deepest divergences in land plants inferred from phylogenomic evidence. Proceedings of the National Academy of Sciences of the United States of America, 103, 15511-15516. 
Rensing, S.A., Goffinet, B., Meyberg, R., Wu, S.Z. and Bezanilla, M. (2020) The Moss Physcomitrium (Physcomitrella) patens: A Model Organism for Non-Seed Plants. Plant Cell, 32, 1361-1376.

Rensing, S.A., Lang, D., Zimmer, A.D., Terry, A., Salamov, A., Shapiro, H., Nishiyama, T., Perroud, P.F., Lindquist, E.A., Kamisugi, Y., Tanahashi, T., Sakakibara, K., Fujita, T., Oishi, K., Shin, I.T., Kuroki, Y., Toyoda, A., Suzuki, Y., Hashimoto, S., Yamaguchi, K., Sugano, S., Kohara, Y., Fujiyama, A., Anterola, A., Aoki, S., Ashton, N., Barbazuk, W.B., Barker, E., Bennetzen, J.L., Blankenship, R., Cho, S.H., Dutcher, S.K., Estelle, M., Fawcett, J.A., Gundlach, H., Hanada, K., Heyl, A., Hicks, K.A., Hughes, J., Lohr, M., Mayer, K., Melkozernov, A., Murata, T., Nelson, D.R., Pils, B., Prigge, M., Reiss, B., Renner, T., Rombauts, S., Rushton, P.J., Sanderfoot, A., Schween, G., Shiu, S.H., Stueber, K., Theodoulou, F.L., Tu, H., Van de Peer, Y., Verrier, P.J., Waters, E., Wood, A., Yang, L., Cove, D., Cuming, A.C., Hasebe, M., Lucas, S., Mishler, B.D., Reski, R., Grigoriev, I.V., Quatrano, R.S. and Boore, J.L. (2008) The Physcomitrella genome reveals evolutionary insights into the conquest of land by plants. Science, 319, 64-69.

Rudinger, M., Szovenyi, P., Rensing, S.A. and Knoop, V. (2011) Assigning DYW-type PPR proteins to RNA editing sites in the funariid mosses Physcomitrella patens and Funaria hygrometrica. Plant J., 67, 370-380.

Scheibe, R. (2004) Malate valves to balance cellular energy supply. Physiol. Plant., 120, 2126.

Scheibe, R., Backhausen, J.E., Emmerlich, V. and Holtgrefe, S. (2005) Strategies to maintain redox homeostasis during photosynthesis under changing conditions. J. Exp. Bot., 56, 1481-1489.

Schilling, B., Christensen, D., Davis, R., Sahu, A.K., Hu, L.I., Walker-Peddakotla, A., Sorensen, D.J., Zemaitaitis, B., Gibson, B.W. and Wolfe, A.J. (2015) Protein acetylation dynamics in response to carbon overflow in Escherichia coli. Mol. Microbiol., 98, 847-863.

Shameer, S., Ratcliffe, R.G. and Sweetlove, L.J. (2019) Leaf Energy Balance Requires Mitochondrial Respiration and Export of Chloroplast NADPH in the Light. Plant Physiol., 180, 1947-1961.

Shore, J.D. and Chakrabarti, S.K. (1976) Subunit dissociation of mitochondrial malate dehydrogenase. Biochemistry, 15, 875-879.

Smith-Hammond, C.L., Hoyos, E. and Miernyk, J.A. (2014) The pea seedling mitochondrial N(epsilon)-lysine acetylome. Mitochondrion, 19 Pt B, 154-165.

Strotbek, C., Krinninger, S. and Frank, W. (2013) The moss Physcomitrella patens: methods and tools from cultivation to targeted analysis of gene function. Int. J. Dev. Biol., 57, 553-564.

Sweetlove, L.J., Beard, K.F., Nunes-Nesi, A., Fernie, A.R. and Ratcliffe, R.G. (2010) Not just a circle: flux modes in the plant TCA cycle. Trends Plant Sci., 15, 462-470.

Tamura, Y., Ito, A. and Cresswell, A.G. (2015) A systematic muscle model covering regions from the fast ramp stretches in the muscle fibres to the relatively slow stretches in the human triceps surae. Comput Methods Biomech Biomed Engin, 18, $97-$ 106.

Tomaz, T., Bagard, M., Pracharoenwattana, I., Linden, P., Lee, C.P., Carroll, A.J., Stroher, E., Smith, S.M., Gardestrom, P. and Millar, A.H. (2010) Mitochondrial malate dehydrogenase lowers leaf respiration and alters photorespiration and plant growth in Arabidopsis. Plant Physiol., 154, 1143-1157.

Trifinopoulos, J., Nguyen, L.T., von Haeseler, A. and Minh, B.Q. (2016) W-IQ-TREE: a fast online phylogenetic tool for maximum likelihood analysis. Nucleic Acids Res., 44, W232-235. 
Tronconi, M.A., Fahnenstich, H., Gerrard Weehler, M.C., Andreo, C.S., Flugge, U.I., Drincovich, M.F. and Maurino, V.G. (2008) Arabidopsis NAD-malic enzyme functions as a homodimer and heterodimer and has a major impact on nocturnal metabolism. Plant Physiol., 146, 1540-1552.

Tronconi, M.A., Hüdig, M., Schranz, M.E. and Maurino, V.G. (2020) Independent recruitment of duplicated $\beta$-subunit-coding NAD-ME genes aided the evolution of C4 photosynthesis in Cleomaceae. Front. Plant Sci.

Venkat, S., Gregory, C., Sturges, J., Gan, Q. and Fan, C. (2017) Studying the Lysine Acetylation of Malate Dehydrogenase. J. Mol. Biol., 429, 1396-1405.

Ventura, M., Mateo, F., Serratosa, J., Salaet, I., Carujo, S., Bachs, O. and Pujol, M.J. (2010) Nuclear translocation of glyceraldehyde-3-phosphate dehydrogenase is regulated by acetylation. Int J Biochem Cell Biol, 42, 1672-1680.

Wang, Q., Zhang, Y., Yang, C., Xiong, H., Lin, Y., Yao, J., Li, H., Xie, L., Zhao, W., Yao, Y., Ning, Z.B., Zeng, R., Xiong, Y., Guan, K.L., Zhao, S. and Zhao, G.P. (2010) Acetylation of metabolic enzymes coordinates carbon source utilization and metabolic flux. Science, 327, 1004-1007.

Weinert, B.T., Iesmantavicius, V., Moustafa, T., Scholz, C., Wagner, S.A., Magnes, C., Zechner, R. and Choudhary, C. (2014) Acetylation dynamics and stoichiometry in Saccharomyces cerevisiae. Mol. Syst. Biol., 10, 716.

Weinert, B.T., Wagner, S.A., Horn, H., Henriksen, P., Liu, W.R., Olsen, J.V., Jensen, L.J. and Choudhary, C. (2011) Proteome-wide mapping of the Drosophila acetylome demonstrates a high degree of conservation of lysine acetylation. Science signaling, $\mathbf{4}$, ra48.

Xia, P., Wang, Z., Liu, X., Wu, B., Wang, J., Ward, T., Zhang, L., Ding, X., Gibbons, G., Shi, Y. and Yao, X. (2012) EB1 acetylation by P300/CBP-associated factor (PCAF) ensures accurate kinetochore-microtubule interactions in mitosis. Proceedings of the National Academy of Sciences of the United States of America, 109, 16564-16569.

Xiong, Y., Peng, X., Cheng, Z., Liu, W. and Wang, G.L. (2016) A comprehensive catalog of the lysine-acetylation targets in rice (Oryza sativa) based on proteomic analyses. Journal of proteomics, 138, 20-29.

Xu, H., Zhang, J., Zeng, J., Jiang, L., Liu, E., Peng, C., He, Z. and Peng, X. (2009) Inducible antisense suppression of glycolate oxidase reveals its strong regulation over photosynthesis in rice. J. Exp. Bot., 60, 1799-1809.

Yang, X.J. and Seto, E. (2008) Lysine acetylation: codified crosstalk with other posttranslational modifications. Mol. Cell, 31, 449-461.

Yoshida, K. and Hisabori, T. (2016) Adenine nucleotide-dependent and redox-independent control of mitochondrial malate dehydrogenase activity in Arabidopsis thaliana. Biochim. Biophys. Acta, 1857, 810-818.

Zhang, M., Chen, G.X., Lv, D.W., Li, X.H. and Yan, Y.M. (2015) N-linked glycoproteome profiling of seedling leaf in Brachypodium distachyon L. Journal of proteome research, 14, 1727-1738.

Zhang, Y., Beard, K.F.M., Swart, C., Bergmann, S., Krahnert, I., Nikoloski, Z., Graf, A., Ratcliffe, R.G., Sweetlove, L.J., Fernie, A.R. and Obata, T. (2017) Protein-protein interactions and metabolite channelling in the plant tricarboxylic acid cycle. Nature communications, 8, 15212.

Zhang, Y., Swart, C., Alseekh, S., Scossa, F., Jiang, L., Obata, T., Graf, A. and Fernie, A.R. (2018) The Extra-Pathway Interactome of the TCA Cycle: Expected and Unexpected Metabolic Interactions. Plant Physiol., 177, 966-979.

Zhao, S., Xu, W., Jiang, W., Yu, W., Lin, Y., Zhang, T., Yao, J., Zhou, L., Zeng, Y., Li, H., Li, Y., Shi, J., An, W., Hancock, S.M., He, F., Qin, L., Chin, J., Yang, P., 
Chen, X., Lei, Q., Xiong, Y. and Guan, K.L. (2010) Regulation of cellular metabolism by protein lysine acetylation. Science, 327, 1000-1004.

Zhen, S., Deng, X., Wang, J., Zhu, G., Cao, H., Yuan, L. and Yan, Y. (2016) First Comprehensive Proteome Analyses of Lysine Acetylation and Succinylation in Seedling Leaves of Brachypodium distachyon L. Scientific reports, $\mathbf{6}, 31576$.

Zhou, H., Finkemeier, I., Guan, W., Tossounian, M.A., Wei, B., Young, D., Huang, J., Messens, J., Yang, X., Zhu, J., Wilson, M.H., Shen, W., Xie, Y. and Foyer, C.H. (2018) Oxidative stress-triggered interactions between the succinyl- and acetylproteomes of rice leaves. Plant Cell Environ, 41, 1139-1153. 
Table 1. Conservation of lysine acetylation between mitochondrial TCA cycle and PDC proteins identified in A. thaliana and P. patens.

\begin{tabular}{|c|c|c|c|c|c|c|}
\hline \multirow[b]{2}{*}{$\begin{array}{l}\text { Orthologous } \\
\text { protein group }\end{array}$} & \multirow[b]{2}{*}{ Accession No. } & \multicolumn{4}{|c|}{$\begin{array}{r}\text { Number of acetylated } \\
\text { lysines }\end{array}$} & \multirow[b]{2}{*}{$\begin{array}{l}\text { Position(s) of } \\
\operatorname{acK}(s)\end{array}$} \\
\hline & & total & diff & $\begin{array}{l}\text { shared } \\
\text { K }\end{array}$ & $\begin{array}{l}\text { shared } \\
\text { acK }\end{array}$ & \\
\hline \multirow{4}{*}{$\begin{array}{l}\text { Pyruvate } \\
\text { dehydrogenase } \\
\text { complex E1 } \alpha\end{array}$} & AT1G24180.1 & 2 & 2 & 1 & 1 & $\underline{\mathbf{8 5}} ; 336$ \\
\hline & AT1G59900.1 & 2 & & & & $\overline{\mathbf{8 1}} ; 332$ \\
\hline & Pp3c12_7580V3.1 & 1 & & & & \\
\hline & Pp3c4_20210V3.1 & 1 & & & & $\overline{86}$ \\
\hline \multirow[t]{5}{*}{ Aconitase (TCA) } & AT2G05710.1 & 3 & 7 & 5 & 1 & $\underline{\mathbf{7 9 0}} ; 846 ; 895$ \\
\hline & AT4G26970.1 & 2 & & & & $\overline{\mathbf{7 9 5}} ; 900$ \\
\hline & Pp3c15_25170V3.1 & 5 & & & & $\overline{520} ; 529 ; 598 ; \underline{8}$ \\
\hline & & & & & & $\overline{\mathbf{0 3}} ; 844$ \\
\hline & Pp3c9_25990V3.2 & 2 & & & & \\
\hline \multirow{3}{*}{$\begin{array}{l}\text { 2-oxoglutarate } \\
\text { dehydrogenase E1 } \\
\text { (TCA) }\end{array}$} & AT3G55410.1 & 3 & 5 & 2 & 0 & $549 ; \underline{599 ; 613}$ \\
\hline & AT5G65750.1 & 2 & & & & $\underline{603 ; 873}$ \\
\hline & Pp3c23_7750V3.1 & 1 & & & & $\overline{1000}$ \\
\hline \multirow{4}{*}{$\begin{array}{l}\text { NAD isocitrate } \\
\text { dehydrogenase } \\
\text { (TCA) }\end{array}$} & AT3G09810.1 & 1 & 3 & 3 & 0 & $\underline{181}$ \\
\hline & AT5G03290.1 & 2 & & & & $\underline{181 ; 218}$ \\
\hline & Pp3c15_3920V3.1 & 1 & & & & 365 \\
\hline & Pp3c9_4370V3.1 & 1 & & & & $\underline{355}$ \\
\hline \multirow{6}{*}{$\begin{array}{l}\text { Malate } \\
\text { dehydrogenase } \\
\text { (TCA) }\end{array}$} & $\begin{array}{l}\text { AT1G53240.1/mMD } \\
\text { H1 }\end{array}$ & 4 & 5 & 3 & 0 & $\underline{170 ; 325 ; 329 ; 3}$ \\
\hline & AT3G15020.1/mMDH & & & & & \\
\hline & 2 & 1 & & & & $\underline{170}$ \\
\hline & Pp3c4_20940V3.1/M & 1 & & & & $\underline{172}$ \\
\hline & DH1 & & & & & \\
\hline & $\begin{array}{l}\text { Pp3c12_8120V3.1/m } \\
\text { MDH2 }\end{array}$ & 1 & & & & $\underline{170}$ \\
\hline Sum & AT: 10/Pp: 9 & 36 & 22 & 14 & 2 & \\
\hline
\end{tabular}

Note: Proteins identified to be lysine-acetylated in P. patens and A. thaliana are organized into orthologous groups. Total numbers of identified acetylated lysines (K, total), number of acetylated Ks at different positions in the orthologous proteins (diff), numbers of acetylated Ks with conserved lysines at same position of at least one putative ortholog of the other species (shared $\mathrm{K}$ ) and shared acetylated lysines (shared acK) are listed separately. Positions of the acetylated Ks (acK) in the proteins are given and numbers are underlined, when Ks are conserved in the putative orthologs at this position. Bold numbers represent acetyated lysines, which were detected in at least one P. patens and one A. thaliana putative ortholog of both datasets. The malate dehydrogenase proteins shown in red are further assessed in this study. Pp: P. patens, AT: A. thaliana. 


\section{Figure Legends}

Figure 1. Direction of the mMDH reaction (red arrow) depending on the metabolic demands of the cells and the NAD redox state of the mitochondrial matrix. A. Catabolic mode of the TCA cycle. B. Provision of OAA for the synthesis of citrate as precursor for nitrogen assimilation. C. Provision of OAA for the synthesis of aspartate. D. Provision of $\mathrm{NAD}^{+}$for the photorespiratory glycine decarboxylase (GDC) and of malate for redox balance between matrix and cytosol. (Modified from Sweetlove et al., 2010).

Figure 2: Distribution of lysine acetylation in $\boldsymbol{P}$. patens proteins. Displayed are the proteins identified in gametophore samples of $P$. patens (left) and the proteins with detected acetylation sites (right). The proteins are classified as mitochondrial (red), plastidial (green), other compartment (blue) and non-annotated (grey) based on the localization identified in Müller et al. (2014) and the annotation provided by Lang et al. (2018). Proteins which could only be classified as mitochondrial or plastidial are displayed in dark red. Manually inspection suggested different localisation for 18 proteins; modified numbers are given in brackets. Organellar proteins are overrepresented in the lysine acetylation dataset ( $\mathrm{p}$-value $<0,0001$ for equal distribution of acetylation in organellar proteins and non-organellar proteins, statistical significance was tested with chi-square test using Excel package, XLSTAT (Addinsoft)).

Figure 3. Sequence conservation of mMDH of representative species of each main land plant clade, human and $\boldsymbol{E}$. coli, and their lysine acetylation sites. Alignment of the acetylated protein isoforms of the mMDHs of the angiosperms A. thaliana, F. ananassa, P. sativum, V. vinifera, $C$. sinensis, $O$. sativa and $B$. distachyon with found lysine acetylation sites marked in blue. Orthologues of representative species of other major land plant clades (the ferns A. filiculoides and S. cucullata, the hornwort A. agrestis, the moss $P$. patens, the liverwort $M$. polymorpha and the green algae $C$. reinhardtii) share high sequence conservation. Shading in black indicates $100 \%$, in dark grey $>80 \%$ and in light grey $>60 \%$ amino acid identity conservation. The cladogram is based on the current understanding of land plant phylogeny (Qiu et al., 2006, Bremer et al., 2009). The sequences of $H$. sapiens mitochondrial MDH2 and E. coli $\mathrm{MDH}$ with their identified acetylated lysines (blue) are included for comparison. The numbering is based on the sequence of MDH1 of A. thaliana. The alignment starts from amino acid 30 which corresponds to the start methionine of E. coli $\mathrm{MDH}$. Yellow highlighted Ks are the acetylated lysines in A. thaliana and P. patens mMDH1 analyzed in this study. A region with acetylation sites conserved in different species is framed in orange and includes position K169 and K170 marked with black arrows.

Figure 4. The impact of lysine acetylation on mMDH activity. A. SDS-PAGE stained with Coomassie of the isolated recombinant non-modified (denoted as WT) and lysine acetylated (AcK) variants of $P$. patens $(P p)$ and A. thaliana (At) mMDH1. To the left, molecular weight markers (Spectra Multicolor Broad Range Protein Ladder; ThermoFisher Scientific). B. Kinetics parameters of the reduction of 
OAA and oxidation of malate by recombinant non-modified (denoted as WT) and AcK versions of $A$. thaliana and $P$. patens mMDH1. Data were adjusted to Michaelis-Menten equation by non-lineal regression with Prism 6 (GraphPad Software). The values represent the mean \pm standard deviation; $\mathrm{n}=$ at least three independent enzyme preparations, each measured in triplicate. * denotes values that are are statistically significantly different from the corresponding WT evaluated by the unpaired t-test. The p-values obtained are indicated in brackets under the evaluated values. C. Native PAGE of recombinant non-modified (WT) and lysine-acetylated (AcK) variants of $P$. patens $(P p)$ and $A$. thaliana (At) mMDH1. The position of the molecular weight markers (Sigma Aldrich) B-amlyase (200 $\mathrm{kDa})$, alcohol dehydrogenase ( $150 \mathrm{kDa})$ and bovine serum albumin $(66 \mathrm{kDa})$ are shown in the left.

Figure 5. Structures of $A$. thaliana and $P$. patens $\mathbf{m M D H 1}$ as obtained by homology modelling. Structures of A. thaliana (bright green A, C, E) and P. patens (dark green; B, D, F) mMDH1 modelled as monomers ( $\mathrm{A}$ and $\mathrm{B})$, dimers (B and $\mathrm{D})$, and tetramers (E and $\mathrm{F})$. The structures were modelled using the crystal structure of $E$. coli $\mathrm{MDH}$ (PDB ID:1EMD) and human MDH2 (PDB ID: 2DFD). The acetylated lysine residues of $A$. thaliana mMDH1 are shown in magenta. The acetylated lysine residue of $P$. patens $\mathrm{mMDH} 1$ is shown in blue. In $\mathrm{A}$ and $\mathrm{B}$, the ligand molecules $\mathrm{NAD}^{+}$and citrate - in brown - were manually fitted in by aligning the structure of the H. sapiens $\mathrm{MDH} 2$. Dotted lines indicate the dimer and tetramer interfaces.

Figure 6. Superposition analysis of MDH monomeric structures. A. Structures of A. thaliana (bright green-magenta) and P. patens (dark green-blue) mMDH1. B. Structures of A. thaliana and $P$. patens $\mathrm{mMDH} 1$, and E. coli $\mathrm{MDH}$ (gold-red; PDB ID:1EMD). C. Zoom in of the region containing K170 in A. thaliana (bright green-magenta) mMDH1, K172 in P. patens (dark green-blue) mMDH1, and K140 in E. coli (gold-red) MDH. D. Zoom in of the carboxy-terminal alpha helix containing K329 and $\mathrm{K} 334$ in A. thaliana (bright green-magenta) mMDH1 and K300 and K301 in E. coli (gold-red) MDH. E. A. thaliana and $P$. patens mMDH1, and human MDH2 (rose-brown; PDB ID: 2DFD). The acetylated lysine residues are shown in magenta for A. thaliana mMDH1, blue for P. patens mMDH1 red for E. coli $\mathrm{MDH}$, and brown for human $\mathrm{MDH}$. For simplicity not all lysines found to be acetylated in E. coli are numbered. 
A

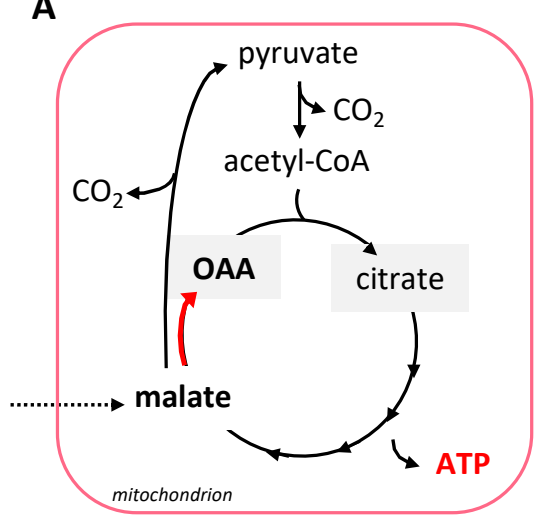

C

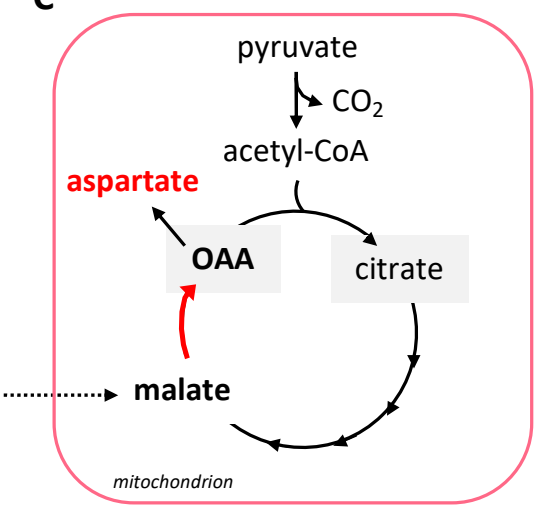

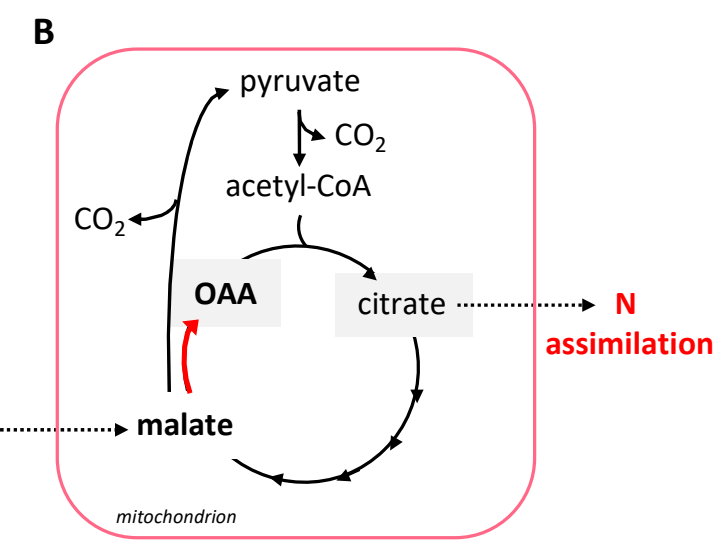

D

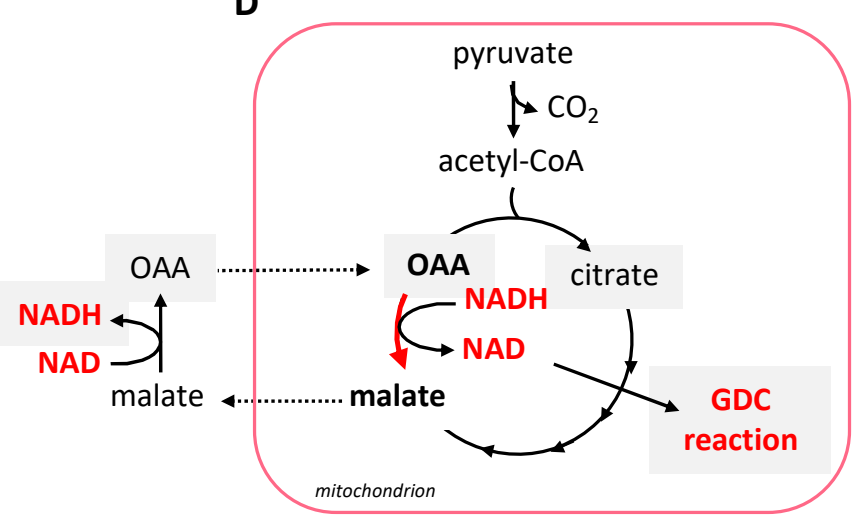

Figure 1. Direction of the mMDH reaction (red arrow) depending on the metabolic demands of the cells and the NAD redox state of the mitochondrial matrix. A. Catabolic mode of the TCA cycle. B. Provision of OAA for the synthesis of citrate as precursor for nitrogen assimilation. C. Provision of OAA for the synthesis of aspartate. D. Provision of $\mathrm{NAD}^{+}$for the photorespiratory glycine decarboxylase (GDC) and of malate for redox balance between matrix and cytoplasm. (Modified from Sweetlove et al., 2010). 


\section{Physcomitrium patens gametophores}

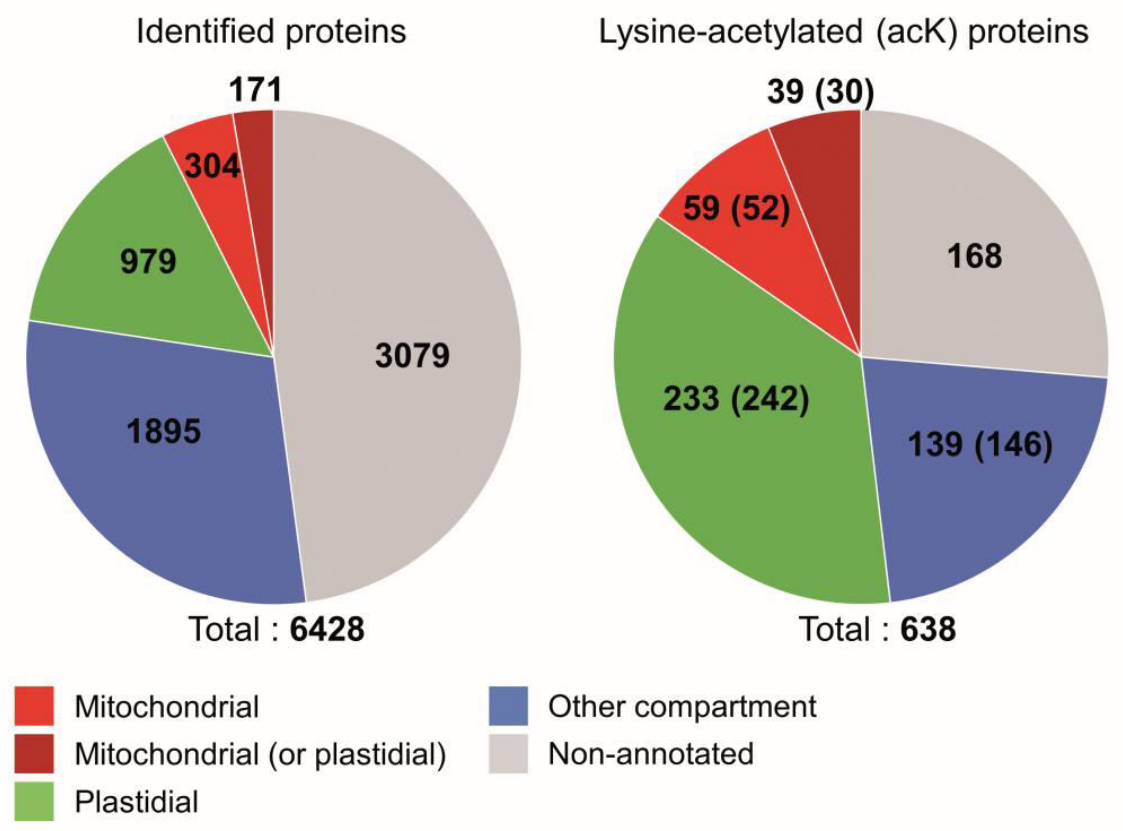

Figure 2: Distribution of lysine acetylation in $\boldsymbol{P}$. patens proteins. Displayed are the proteins identified in gametophore samples of P. patens (left) and the proteins with detected acetylation sites (right). The proteins are classified as mitochondrial (red), plastidial (green), other compartment (blue) and non-annotated (grey) based on the localization identified in Müller et al. (2014) and the annotation provided by Lang et al. (2018). Proteins which could only be classified as mitochondrial or plastidial are displayed in dark red. Manually inspection suggested different localisation for 18 proteins; modified numbers are given in brackets. Organellar proteins are overrepresented in the lysine acetylation dataset ( $\mathrm{p}$-value $<0.0001$ for equal distribution of acetylation in organellar proteins and non-organellar proteins, statistical significance was tested with chi-square test using Excel package, XLSTAT (Addinsoft)). 


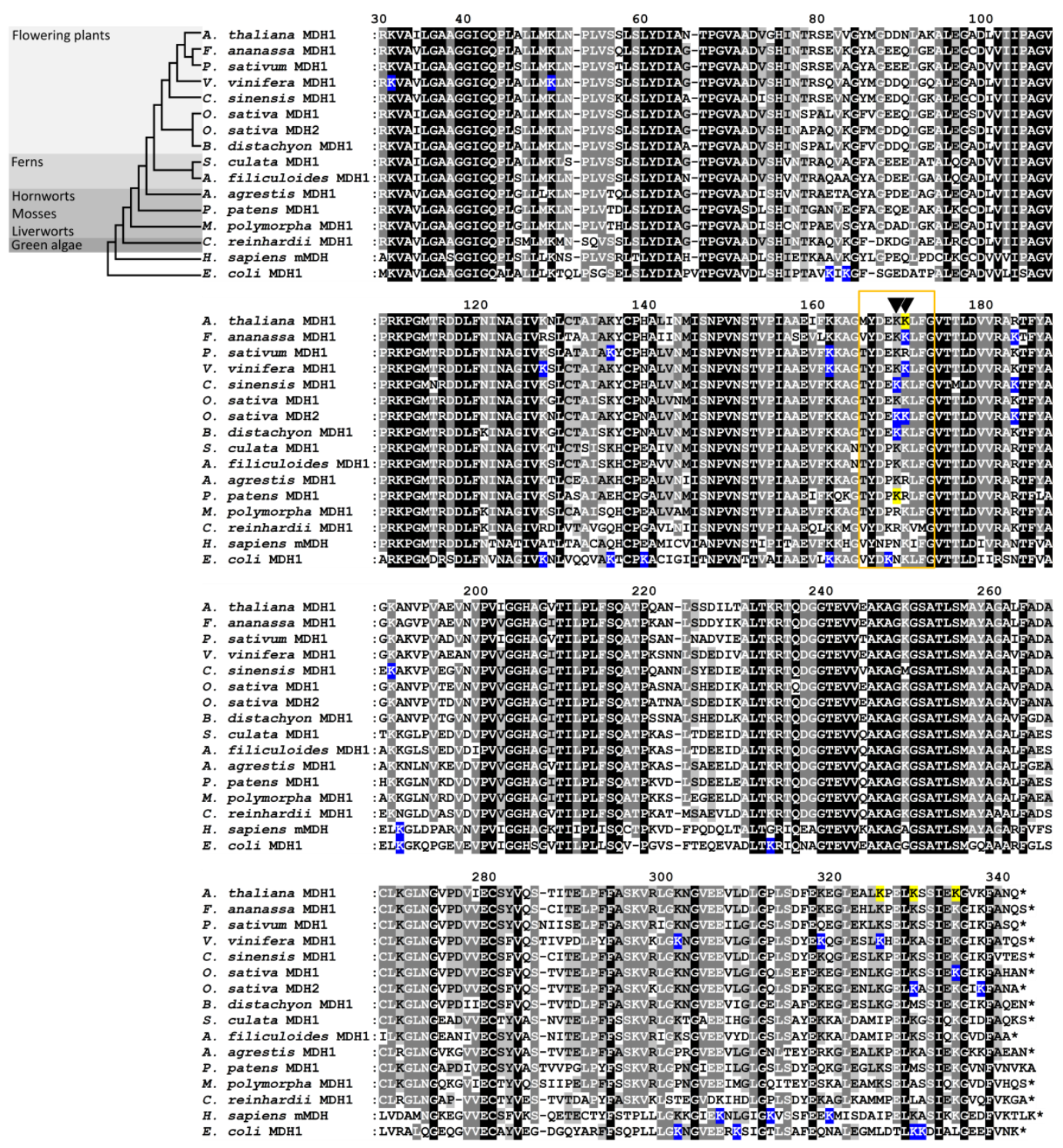

Figure 3. Sequence conservation of mMDH of representative species of each main land plant clade, human and $\boldsymbol{E}$. coli, and their lysine acetylation sites. Alignment of the acetylated protein isoforms of the mMDHs of the angiosperms A. thaliana, $F$. ananassa, $P$. sativum, V. vinifera, $C$. sinensis, $O$. sativa and $B$. distachyon with found lysine acetylation sites marked in blue. Orthologues of representative species of other major land plant clades (the ferns A. filiculoides and S. cucullata, the hornwort A. agrestis, the moss P. patens, the liverwort M. polymorpha and the green algae $C$. reinhardtii) share high sequence conservation. Shading in black indicates $100 \%$, in dark grey $>80 \%$ and in light grey $>60 \%$ amino acid identity conservation. The cladogram is based on the current understanding of land plant phylogeny (Qiu et al., 2006, Bremer et al., 2009). The sequences of $H$. sapiens mitochondrial $\mathrm{MDH} 2$ and E. coli $\mathrm{MDH}$ with their identified acetylated lysines (blue) are included for comparison. The numbering is based on the sequence of MDH1 of A. thaliana. The alignment starts from amino acid 30 which corresponds to the start methionine of E. coli MDH. Yellow highlighted Ks are the acetylated lysines in A. thaliana and P. patens mMDH1 analyzed in this study. A region with acetylation sites conserved in different species is framed in orange and includes position K169 and K170 marked with black arrows. 

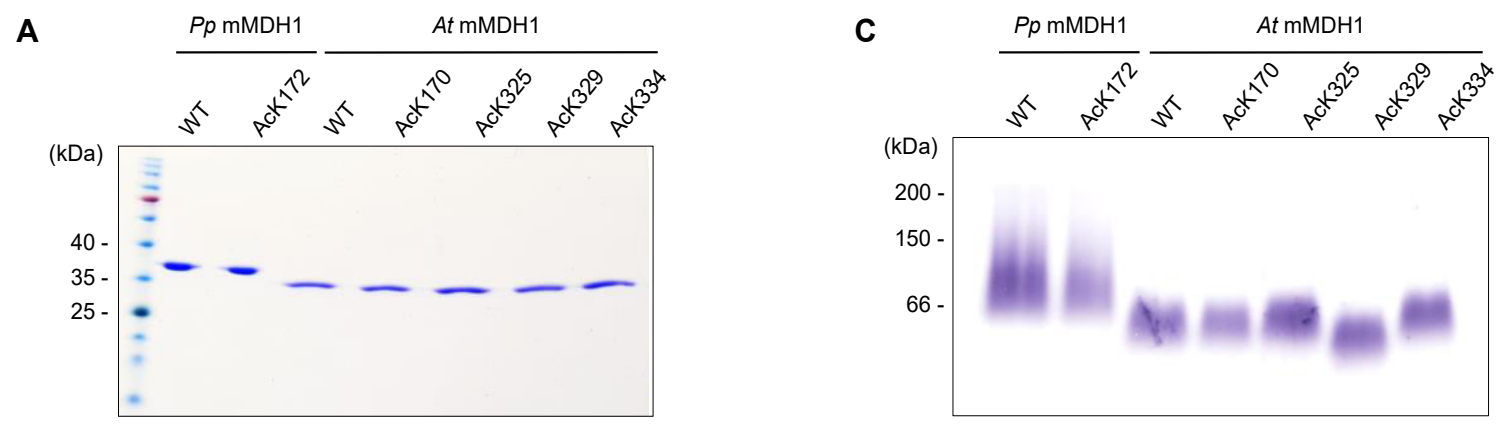

B

\begin{tabular}{|c|c|c|c|c|c|c|c|c|c|}
\hline & & \multicolumn{6}{|c|}{ A. thaliana } & \multicolumn{2}{|c|}{ P. patens } \\
\hline & & WT & AcK169 & AcK170 & AcK325 & AcK329 & AcK334 & WT & AcK172 \\
\hline \multirow{6}{*}{$\begin{array}{l}\frac{\Phi}{\pi} \\
\frac{\pi}{\pi} \\
\frac{\varepsilon}{\uparrow} \\
\uparrow \\
\frac{\delta}{\delta} \\
0\end{array}$} & $K_{m} \mathrm{OAA}(\mu \mathrm{M})$ & $17 \pm 7$ & $39 \pm 8^{*}$ & $9 \pm 3^{*}$ & $20 \pm 12$ & $17 \pm 4$ & $18 \pm 6$ & $18 \pm 8$ & $23 \pm 11$ \\
\hline & (P-value) & & $(<0.0001)$ & $(0.006)$ & $(0.526)$ & $(1.000)$ & $(0.749)$ & & $(0.286)$ \\
\hline & $k_{\text {cat }}\left(\mathrm{s}^{-1}\right)$ & $548 \pm 111$ & $582 \pm 12$ & $204 \pm 33^{*}$ & $471 \pm 208$ & $484 \pm 130$ & $353 \pm 18^{*}$ & $271 \pm 17$ & $547 \pm 41^{*}$ \\
\hline & (P-value) & & $(0.375)$ & $(<0.0001)$ & $(0.342)$ & $(0.278)$ & $(<0.0001)$ & & $(<0.0001)$ \\
\hline & $k_{\text {cat }} / K_{m}\left(\mu \mathrm{M}^{-1} \mathrm{~s}^{-1}\right)$ & $38.8 \pm 19.0$ & $15 \pm 6^{*}$ & $24.1 \pm 10.6$ & $28.4 \pm 12.5$ & $29.1 \pm 3.8$ & $24.9 \pm 7.4$ & $14.8 \pm 2.3$ & $23.7 \pm 3.7^{*}$ \\
\hline & (P-value) & & $(0.003)$ & $(0.065)$ & $(0.205)$ & $(0.168)$ & $(0.064)$ & & $(<0.0001)$ \\
\hline \multirow{6}{*}{$\begin{array}{l}\frac{\pi}{\delta} \\
0 \\
\uparrow \\
\frac{0}{ \pm} \\
\frac{\pi}{\pi} \\
\varepsilon\end{array}$} & $K_{m}$ malate $(\mu \mathrm{M})$ & $298 \pm 9$ & $308 \pm 30$ & $492 \pm 44^{*}$ & $253 \pm 14^{*}$ & $226 \pm 34^{*}$ & $78 \pm 37^{*}$ & $384 \pm 50$ & $354 \pm 49$ \\
\hline & (P-value) & & $(0.088)$ & $(<0.0001)$ & $(<0.0001)$ & $(<0.0001)$ & $(<0.0001)$ & & $(0.175)$ \\
\hline & $k_{\text {cat }}\left(\mathrm{s}^{-1}\right)$ & $118 \pm 16$ & $119 \pm 2$ & $132 \pm 18$ & $94 \pm 7^{*}$ & $90 \pm 13^{*}$ & $93 \pm 21$ & $107 \pm 14$ & $111 \pm 23$ \\
\hline & (P-value) & & $(0.855)$ & $(0.100)$ & $(0.001)$ & $(0.001)$ & $(0.068)$ & & $(0.662)$ \\
\hline & $k_{\text {cat }} / K_{m}\left(\mu \mathrm{M}^{-1} \mathrm{~s}^{-1}\right)$ & $0.40 \pm 0.04$ & $0.38 \pm 0.07$ & $0.27 \pm 0.05^{*}$ & $0.37 \pm 0.05$ & $0.40 \pm 0.08$ & $1.34 \pm 0.56^{*}$ & $0.28 \pm 0.08$ & $0.32 \pm 0.07$ \\
\hline & (P-value) & & $(0.468)$ & $(<0.0001)$ & $(0.261)$ & $(0.845)$ & $(0.0001)$ & & $(0.343)$ \\
\hline
\end{tabular}

Figure 4. The impact of lysine acetylation on mMDH activity. A. SDS-PAGE stained with Coomassie of the isolated recombinant non-modified (denoted as WT) and lysine acetylated (AcK) variants of $P$. patens $(P p)$ and A. thaliana (At) mMDH1. To the left, molecular weight markers (Spectra Multicolor Broad Range Protein Ladder; ThermoFisher Scientific). B. Kinetics parameters of the reduction of OAA and oxidation of malate by recombinant non-modified (denoted as WT) and AcK versions of $A$. thaliana and $P$. patens mMDH1. Data were adjusted to Michaelis-Menten equation by non-lineal regression with Prism 6 (GraphPad Software). The values represent the mean \pm standard deviation; $\mathrm{n}=$ at least three independent enzyme preparations, each measured in triplicate. * denotes values that are are statistically significantly different from the corresponding WT evaluated by the unpaired t-test. The p-values obtained are indicated in brackets under the evaluated values. C. Native PAGE of recombinant non-modified (WT) and lysine-acetylated (AcK) variants of P. patens $(P p)$ and A. thaliana (At) mMDH1. The position of the molecular weight markers (Sigma Aldrich) B-amlyase (200 $\mathrm{kDa})$, alcohol dehydrogenase $(150 \mathrm{kDa})$ and bovine serum albumin $(66 \mathrm{kDa})$ are shown in the left. 
A

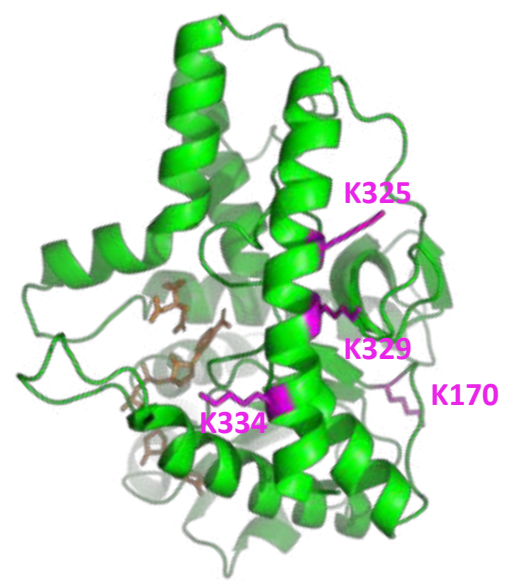

C

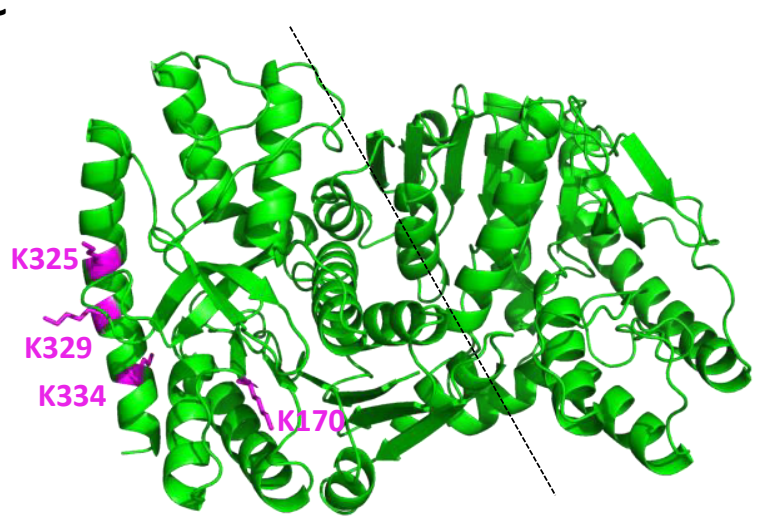

$\mathbf{E}$

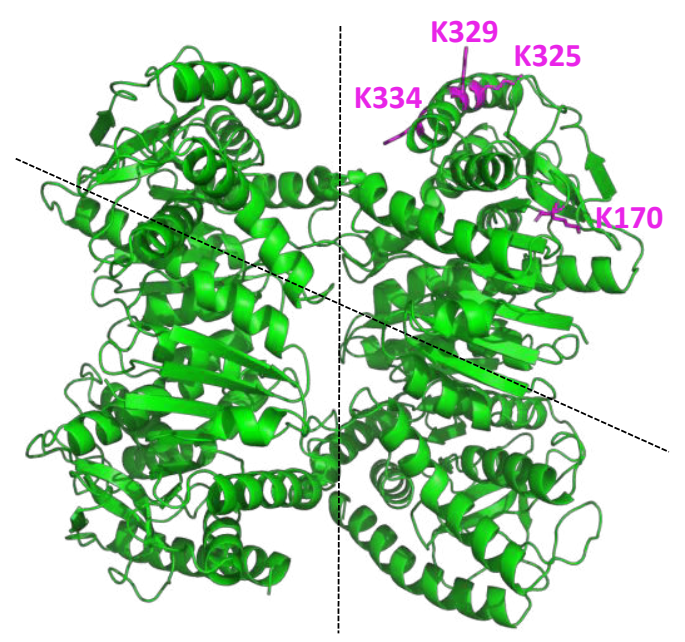

B

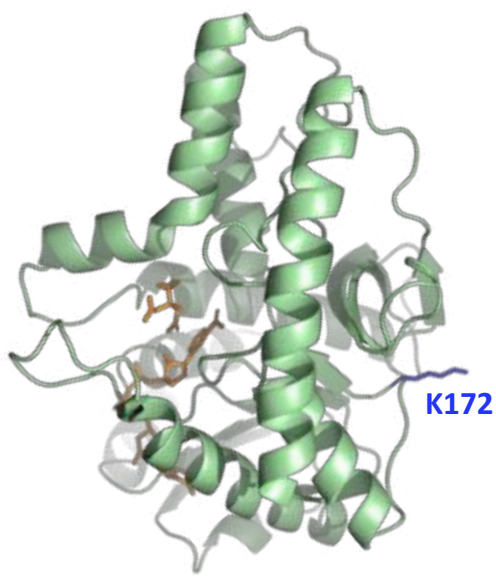

D

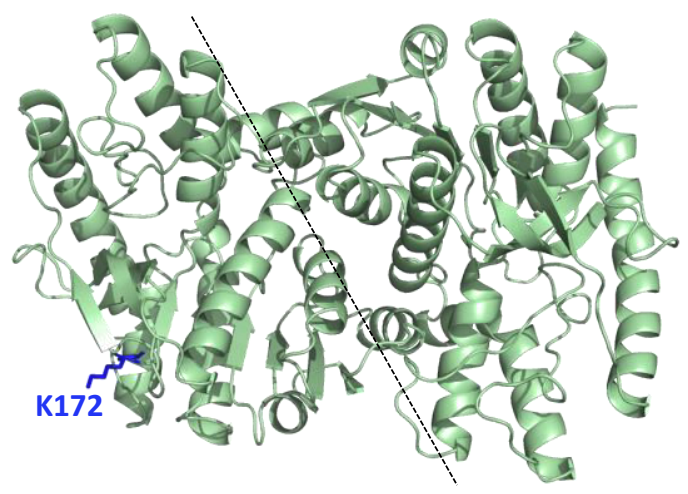

$\mathbf{F}$

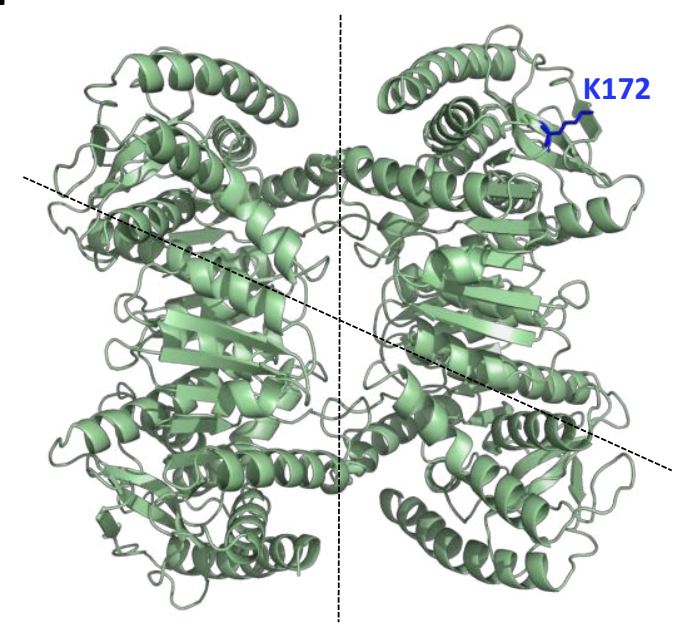

Figure 5. Structures of $A$. thaliana and $P$. patens mMDH1 as obtained by homology modelling. Structures of A. thaliana (bright green A, C, E) and P. patens (dark green; B, D, F) mMDH1 modelled as monomers ( $\mathrm{A}$ and $\mathrm{B})$, dimers (B and $\mathrm{D})$, and tetramers (E and $\mathrm{F})$. The structures were modelled using the crystal structure of $E$. coli MDH (PDB ID:1EMD) and human MDH2 (PDB ID: 2DFD). The acetylated lysine residues of $A$. thaliana mMDH1 are shown in magenta. The acetylated lysine residue of $P$. patens mMDH1 is shown in blue. In A and $\mathrm{B}$, the ligand molecules $\mathrm{NAD}^{+}$and citrate - in brown - were manually fitted in by aligning the structure of the H. sapiens MDH2. Dotted lines indicate the dimer and tetramer interfaces. 
A

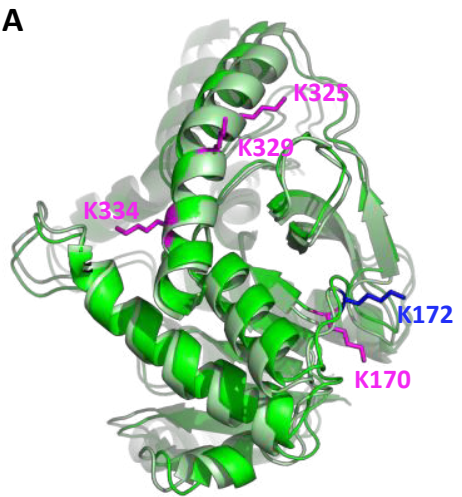

C

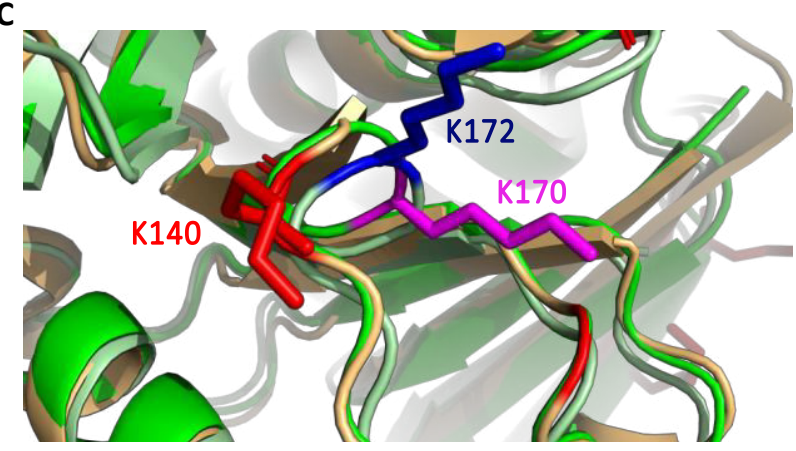

B

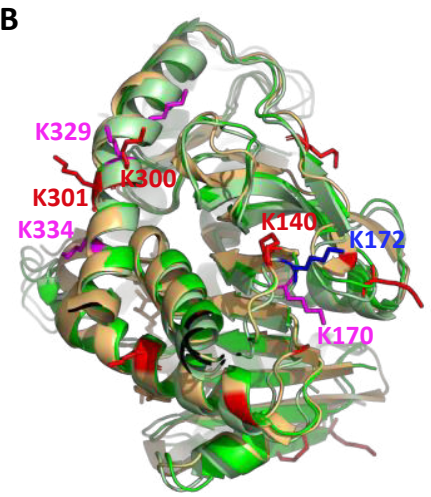

D

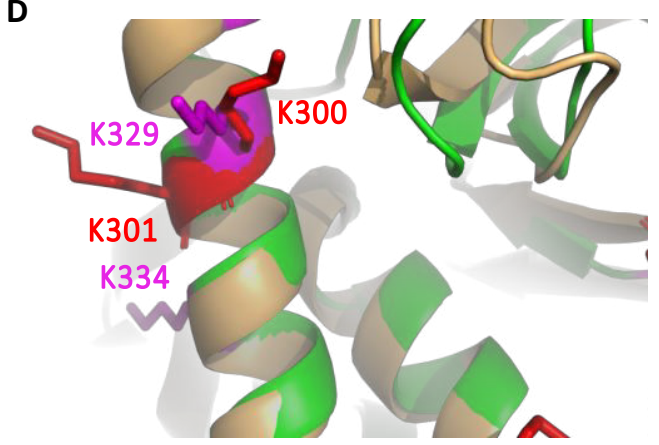

Figure 6. Superposition analysis of MDH monomeric structures. A. Structures of A. thaliana (bright green-magenta) and $P$. patens (dark green-blue) mMDH1. B. Structures of A. thaliana and $P$. patens $\mathrm{mMDH} 1$, and E. coli MDH (gold-red; PDB ID:1EMD). C. Zoom in of the region containing K170 in A. thaliana (bright green-magenta) mMDH1, K172 in P. patens (dark green-blue) mMDH1, and K140 in E. coli (gold-red) MDH. D. Zoom in of the carboxy-terminal alpha helix containing K329 and $\mathrm{K} 334$ in A. thaliana (bright green-magenta) mMDH1 and K300 and K301 in E. coli (gold-red) MDH. E. A. thaliana and P. patens mMDH1, and human MDH2 (rose-brown; PDB ID: 2DFD). The acetylated lysine residues are shown in magenta for A. thaliana mMDH1, blue for P. patens mMDH1 red for E. coli $\mathrm{MDH}$, and brown for human $\mathrm{MDH}$. For simplicity not all lysines found to be acetylated in $E$. coli are numbered. 